\title{
Nonequilibrium entropy limiters in lattice Boltzmann methods
}

\author{
R.A. Brownlee, A.N. Gorban*, J. Levesley \\ Department of Mathematics, University of Leicester, LE1 7RH, UK
}

Received 22 August 2007

Available online 29 September 2007

\begin{abstract}
We construct a system of nonequilibrium entropy limiters for the lattice Boltzmann methods (LBM). These limiters erase spurious oscillations without blurring of shocks, and do not affect smooth solutions. In general, they do the same work for LBM as flux limiters do for finite differences, finite volumes and finite elements methods, but for LBM the main idea behind the construction of nonequilibrium entropy limiter schemes is to transform a field of a scalar quantity — nonequilibrium entropy. There are two families of limiters: (i) based on restriction of nonequilibrium entropy (entropy "trimming") and (ii) based on filtering of nonequilibrium entropy (entropy filtering). The physical properties of LBM provide some additional benefits: the control of entropy production and accurate estimation of introduced artificial dissipation are possible. The constructed limiters are tested on classical numerical examples: 1D athermal shock tubes with an initial density ratio 1:2 and the 2D lid-driven cavity for Reynolds numbers $R e$ between 2000 and 7500 on a coarse $100 \times 100$ grid. All limiter constructions are applicable both for entropic and for non-entropic equilibria.
\end{abstract}

(c) 2007 Elsevier B.V. All rights reserved.

PACS: 47.11.Qr; 47.20.-k; 47.11.-j; 51.10.+y

Keywords: Lattice Boltzmann method; Numerical regularisation; Entropy

\section{Introduction}

In 1959, Godunov [16] demonstrated that a (linear) scheme for a PDE could not, at the same time, be monotone and second-order accurate. Hence, we should choose between spurious oscillation in high order non-monotone schemes and additional dissipation in first-order schemes. Flux limiter schemes are invented to combine high resolution schemes in areas with smooth fields and first-order schemes in areas with sharp gradients.

The idea of flux limiters can be illustrated by computation of the flux $F_{0,1}$ of the conserved quantity $u$ between a cell marked by 0 and one of two its neighbour cells marked by \pm 1 :

$$
F_{0,1}=(1-\phi(r)) f_{0,1}^{\text {low }}+\phi(r) f_{0,1}^{\text {high }},
$$

\footnotetext{
This work was supported by EPSRC grant number GR/S95572/01.

* Corresponding author. Tel.: +44 1162231433 .

E-mail addresses: r.brownlee@mcs.le.ac.uk (R.A. Brownlee), a.gorban@mcs.le.ac.uk (A.N. Gorban), j.levesley@mcs.le.ac.uk (J. Levesley).
} 
where $f_{0,1}^{\text {low }}, f_{0,1}^{\text {high }}$ are low and high resolution scheme fluxes, respectively, $r=\left(u_{0}-u_{-1}\right) /\left(u_{1}-u_{0}\right)$, and $\phi(r) \geq 0$ is a flux limiter function. For $r$ close to 1 , the flux limiter function $\phi(r)$ should be also close to 1 .

Many flux limiter schemes have been invented during the last two decades [39]. No particular limiter works well for all problems, and a choice is usually made on a trial and error basis.

Below are several examples of flux limiter functions:

$$
\begin{aligned}
& \phi_{m m}(r)=\max [0, \min (r, 1)] \quad(\operatorname{minmod}[32]) ; \\
& \phi_{o s}(r)=\max [0, \min (r, \beta)],(1 \leq \beta \leq 2) \quad(\text { Osher [11]); } \\
& \phi_{m c}(r)=\max [0, \min (2 r, 0.5(1+r), 2)] \quad(\text { monotonised central [38]); } \\
& \phi_{s b}(r)=\max [0, \min (2 r, 1), \min (r, 2)] \quad(\text { superbee [32]); } \\
& \phi_{s w}(r)=\max [0, \min (\beta r, 1),(r, \beta)],(1 \leq \beta \leq 2) \quad(\text { Sweby [35]). }
\end{aligned}
$$

The lattice Boltzmann method has been proposed as a discretization of Boltzmann's kinetic equation and is now in wide use in fluid dynamics and beyond (for an introduction and review see Ref. [33]). Instead of fields of moments $M$, the lattice Boltzmann method operates with fields of discrete distributions $f$. This allows us to construct very simple limiters that do not depend on slopes or gradients.

All the limiters we construct are based on the representation of distributions $f$ in the form

$$
f=f^{*}+\left\|f-f^{*}\right\| \frac{f-f^{*}}{\left\|f-f^{*}\right\|},
$$

where $f^{*}$ is the correspondent quasiequilibrium (conditional equilibrium) for given moments $M, f-f^{*}$ is the nonequilibrium "part" of the distribution, which is represented in the form "norm $\times$ direction", and $\left\|f-f^{*}\right\|$ is the norm of that nonequilibrium component (usually this is the entropic norm). Limiters change the norm of the nonequilibrium component $f-f^{*}$, but do not touch its direction or the quasiequilibrium. In particular, limiters do not change the macroscopic variables, because moments for $f$ and $f^{*}$ coincide. All limiters that we use are transformations of the form

$$
f \mapsto f^{*}+\phi \times\left(f-f^{*}\right)
$$

with $\phi>0$. If $f-f^{*}$ is too big, then the limiter should decrease its norm.

The outline of the paper is as follows. In Section 2 we introduce the notions and notation from lattice Boltzmann theory that we need, in Section 3 we elaborate the idea of entropic limiters in more detail and construct several nonequilibrium entropy limiters for LBM, in Section 4 some numerical experiments are described:

(1) 1D athermal shock tube examples;

(2) steady state vortex centre locations and observation of first Hopf bifurcation in 2D lid-driven cavity flow.

In Section 5 we discuss some practical consequences of our tests and estimate additional entropy production and additional viscosity for several cases. Concluding remarks are given in Section 6.

\section{Background}

The essence of lattice Boltzmann methods was formulated by S. Succi in the following maxim: "Nonlinearity is local, non-locality is linear". ${ }^{1}$ We should even strengthen this statement. Non-locality (a) is linear; (b) is exactly and explicitly solvable for all time steps; (c) space discretization is an exact operation.

The lattice Boltzmann method is a discrete velocity method. The finite set of velocity vectors $\left\{\boldsymbol{v}_{i}\right\}(i=1, \ldots, m)$ is selected, and a fluid is described by associating, with each velocity $\boldsymbol{v}_{i}$, a single-particle distribution function $f_{i}=f_{i}(\boldsymbol{x}, t)$ which is evolved by advection and interaction (collision) on a fixed computational lattice. The values $f_{i}$ are named populations. If we look at all lattice Boltzmann models, one finds that there are two steps: free flight for time $\delta t$ and a local collision operation.

\footnotetext{
${ }^{1}$ S. Succi, "Lattice Boltzmann at all-scales: from turbulence to DNA translocation", Mathematical Modelling Centre Distinguished Lecture, University of Leicester, Leicester UK, 15th November 2006.
} 
The free flight transformation for continuous space is

$$
f_{i}(\boldsymbol{x}, t+\delta t)=f_{i}\left(\boldsymbol{x}-\boldsymbol{v}_{i} \delta t, t\right) .
$$

After the free flight step the collision step follows:

$$
f_{i}(\boldsymbol{x}) \mapsto F_{i}\left(\left\{f_{j}(\boldsymbol{x})\right\}\right),
$$

or in the vector form

$$
f(\boldsymbol{x}) \mapsto F(f(\boldsymbol{x})) .
$$

Here, the collision operator $F$ is the set of functions $F_{i}\left(\left\{f_{j}\right\}\right)(i=1, \ldots, m)$. Each function $F_{i}$ depends on all $f_{j}$ $(j=1, \ldots, m)$ : new values of the populations $f_{i}$ at a point $\boldsymbol{x}$ are known functions of all previous population values at the same point.

The lattice Boltzmann chain "free flight $\rightarrow$ collision $\rightarrow$ free flight $\rightarrow$ collision $\ldots b$ " can be exactly restricted onto any space lattice which is invariant with respect to space shifts of the vectors $\boldsymbol{v}_{i} \delta t(i=1, \ldots, c, m)$. Indeed, free flight transforms the population values at sites of the lattice into the population values at sites of the same lattice. The collision operator (2) acts pointwise at each lattice site separately. Much effort has been applied to answering the questions: "How does the lattice Boltzmann chain approximate the transport equation for the moments $M$ ?", and "How does one construct the lattice Boltzmann model for a given macroscopic transport phenomenon?" (a review is presented in book [33]).

In our paper we propose a universal construction of limiters for all possible collision operators, and the detailed construction of $F_{i}\left(\left\{f_{j}\right\}\right)$ is not important for this purpose. The only part of this construction that we use is the local equilibria (sometimes these states are named conditional equilibria, quasiequilibria, or even simpler, equilibria).

The lattice Boltzmann models should describe the macroscopic dynamic, i.e., the dynamic of macroscopic variables. The macroscopic variables $M_{\ell}(\boldsymbol{x})$ are some linear functions of the population values at the same point: $M_{\ell}(\boldsymbol{x})=\sum_{i} m_{\ell i} f_{i}(\boldsymbol{x})$, or in the vector form, $M(\boldsymbol{x})=m(f(\boldsymbol{x}))$. The macroscopic variables are invariants of collisions:

$$
\sum_{i} m_{\ell i} f_{i}=\sum_{i} m_{\ell i} F_{i}\left(\left\{f_{j}\right\}\right) \quad(\text { or } m(f)=m(F(f))) .
$$

The standard example of the macroscopic variables are hydrodynamic fields (density-velocity-energy density): $\{n, n \boldsymbol{u}, E\}(\boldsymbol{x}):=\sum_{i}\left\{1, \boldsymbol{v}_{i}, v_{i}^{2} / 2\right\} f_{i}(\boldsymbol{x})$. But this is not an obligatory choice. On the other hand, the athermal lattice Boltzmann models with a shortened list of macroscopic variables $\{n, n \boldsymbol{u}\}$ are very popular.

The quasiequilibrium is the positive fixed point of the collision operator for the given macroscopic variables $M$. We assume that this point exists, is unique and depends smoothly on $M$. For the quasiequilibrium population vector for given $M$ we use the notation $f_{M}^{*}$, or simply $f^{*}$, if the correspondent value of $M$ is obvious. We use $\Pi^{*}$ to denote the equilibration projection operation of a distribution $f$ into the corresponding quasiequilibrium state:

$$
\Pi^{*}(f)=f_{m(f)}^{*}
$$

For some of the collision models an entropic description of quasiequilibrium is possible: an entropy density function $S(f)$ is defined and the quasiequilibrium point $f_{M}^{*}$ is the entropy maximiser for given $M$ [22,34].

Let the entropy $S(f)$ be defined for each positive population vector $f=\left(f_{i}\right)$ (below we use the same letter $S$ for the entropy local in space and hope that the context will always make this notation clear). We assume that the global entropy on a grid is a sum of local entropies for all sites.

The notion of quasiequilibrium is most general and describes conditional equilibria for any choice of macroscopic variables. If the macroscopic variables are the usual hydrodynamic fields, then for continuous velocity space the quasiequilibria are local Maxwellian, i.e., local equilibria. The same term, local equilibria, is suitable for lattice Boltzmann models too.

As a basic example we consider the lattice Bhatnagar-Gross-Krook (LBGK) model with overrelaxation (see, e.g., Refs. $[4,12,20,24,33])$. The LBGK collision operator is

$$
F(f):=\Pi^{*}(f)+(2 \beta-1)\left(\Pi^{*}(f)-f\right),
$$


where $\beta \in[0,1]$. For $\beta=0$, LBGK collisions do not change $f$, for $\beta=1 / 2$ these collisions act as equilibration (this corresponds to the Ehrenfests' coarse graining [14] further developed in Refs. $[13,18,19]$ ), and for $\beta=1$, LBGK collisions act as a point reflection with the centre at the equilibrium $\Pi^{*}(f)$.

It is shown [8] that under some stability conditions and after an initial period of relaxation, the simplest LBGK collision with overrelaxation $[20,33]$ provides second-order accurate approximation for the macroscopic transport equation with viscosity proportional to $\delta t(1-\beta) / \beta$.

Entropic LBGK (ELBM) methods [5,19,22,34] differ in the definition of (3):

- the quasiequilibrium should be the point of conditional entropy maximum: $S(f) \rightarrow$ max under the condition $m(f)=M$;

- for $\beta=1$ the collision operator should conserve the entropy, and in general has the following form:

$$
F(f):=(1-\beta) f+\beta \tilde{f},
$$

where $\tilde{f}=(1-\alpha) f+\alpha \Pi^{*}(f)$. The number $\alpha=\alpha(f)$ at each space point is chosen so that the local constant entropy condition is satisfied: $S(f)=S(\tilde{f})$. For LBGK $(3), \alpha=2$.

In the low viscosity regime, LBGK suffers from numerical instabilities which readily manifest themselves as local blow-ups and spurious oscillations.

The LBM experiences the same spurious oscillation problems near sharp gradients as high order schemes do. The physical properties of the LBM schemes allow one to construct new types of limiters: the nonequilibrium entropy limiters. In general, they do the same work for LBM as flux limiters do for finite difference, finite volume and finite element methods, but for LBM the main idea behind the construction of nonequilibrium entropy limiter schemes is to limit a scalar quantity - nonequilibrium entropy (and not the vectors or tensors of spatial derivatives, as it is for flux limiters). These limiters introduce some additional dissipation, but all this dissipation could easily be evaluated through analysis of nonequilibrium entropy production.

Two examples of such limiters have been recently proposed: the positivity rule $[6,27,37]$ and the Ehrenfests' regularisation [7]. The positivity rule just provides positivity of distributions: if a collision step produces negative populations, then the positivity rule returns them to the boundary of positivity. In the Ehrenfests' regularisation, one selects the $k$ sites with highest nonequilibrium entropy (the difference between the entropy of the state $f$ and the entropy of the corresponding quasiequilibrium state $f^{*}$ at a given space point) that exceed a given threshold and equilibrates the state at these sites.

The positivity rule and the Ehrenfests' regularisation provide rare, intense and localised corrections. It is easy and also computationally cheap to organise more gentle transformation with smooth shift of highly nonequilibrium states to equilibrium. The following regularisation transformation distributes its action smoothly: we can just choose in (1) $\phi=\phi(\Delta S(f))$ with sufficiently smooth function $\phi(\Delta S(f))$. Here $f$ is the state at some site, $f^{*}$ is the corresponding quasiequilibrium state, $S$ is entropy, and $\Delta S(f):=S\left(f^{*}\right)-S(f)$.

The next step in the development of the nonequilibrium entropy limiters is in the usage of local entropy filters. The filter of choice here is the median filter: it does not erase sharp fronts, and is much more robust than convolution filters.

Not all lattice Boltzmann models are entropic, and an important question arises: "How do we create nonequilibrium entropy limiters for LBM with non-entropic (quasi)equilibria?" We propose a solution of this problem based on the discrete Kullback entropy [25]:

$$
S_{K}(f)=-\sum_{i} f_{i} \ln \left(\frac{f_{i}}{f_{i}^{*}}\right) .
$$

For entropic quasiequilibria with perfect entropy the discrete Kullback entropy gives the same $\Delta S:-S_{K}(f)=$ $\Delta S(f)$. Let the discrete entropy have the standard form for an ideal (perfect) mixture [23]:

$$
S(f)=-\sum_{i} f_{i} \ln \left(\frac{f_{i}}{W_{i}}\right) .
$$




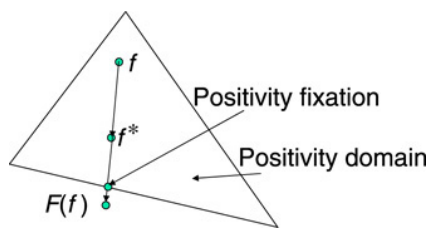

Fig. 1. The positivity rule in action. The motion stops at the positivity boundary.

In quadratic approximation,

$$
-S_{K}(f)=\sum_{i} f_{i} \ln \left(\frac{f_{i}}{f_{i}^{*}}\right) \approx \sum_{i} \frac{\left(f_{i}-f_{i}^{*}\right)^{2}}{f_{i}^{*}} .
$$

If we define $f^{*}$ as the conditional entropy maximum for given $M_{j}=\sum_{k} m_{j k} f_{k}$, then

$$
\ln f_{k}^{*}=\sum_{j} \mu_{j} m_{j k}
$$

where $\mu_{j}(M)$ are the Lagrange multipliers (or "potentials"). For this entropy and conditional equilibrium we find

$$
\Delta S=S\left(f^{*}\right)-S(f)=\sum_{i} f_{i} \ln \left(\frac{f_{i}}{f_{i}^{*}}\right)=-S_{K}(f),
$$

if $f$ and $f^{*}$ have the same moments, $m(f)=m\left(f^{*}\right)$.

In what follows, $\Delta S$ is the Kullback distance $-S_{K}(f)$ (7) for general (positive) quasiequilibria $f^{*}$, or simply $S\left(f^{*}\right)-S(f)$ for entropic quasiequilibria (or second approximations for these quantities (6)).

In thermodynamics, the Kullback entropy belongs to the family of Massieu-Planck-Kramers functions (canonical or grand canonical potentials). There is another sense of this quantity: $S_{K}$ is the relative entropy of $f$ with respect to $f^{*}[17,31]$. We should stress that even in cases when the quasiequilibrium employed is a close approximation of the entropic quasiequilibrium but does not realise the conditional entropy maximum exactly, we have to use the Kullback entropic distance (7) instead of $S\left(f^{*}\right)-S(f)$. The change of definition of $\Delta S$ is necessary to provide positivity of $\Delta S: f^{*}$ always realises the maximum of the Kullback entropy (5) for the given macroscopic variables $M=m(f)$.

\section{Nonequilibrium entropy limiters for LBM}

\subsection{Positivity rule}

There is a simple recipe for positivity preservation [6,27,37]: to substitute nonpositive $F(f)(\boldsymbol{x})$ (3) by the closest nonnegative state that belongs to the straight line

$$
\left\{\lambda f(\boldsymbol{x})+(1-\lambda) \Pi^{*}(f(\boldsymbol{x})) \mid \lambda \in \mathbb{R}\right\}
$$

defined by the two points, $f(\boldsymbol{x})$ and the corresponding quasiequilibrium. This operation is to be applied pointwise, at points of the lattice where positivity is violated. The coefficient $\lambda$ depends on $x$ too. Let us call this recipe the positivity rule (Fig. 1). This recipe preserves positivity of populations and probabilities, but can affect accuracy of approximation. The same rule is necessary for ELBM (4) when the positive "mirror state" $\tilde{f}$ with the same entropy as $f$ does not exist on the straight line (8).

\subsection{The Ehrenfests' regularisation}

For discussing methods with additional dissipation, the entropic approach is very convenient. The local nonequilibrium entropy for each site is

$$
\Delta S(f):=S\left(f^{*}\right)-S(f)
$$

where $f^{*}$ is the corresponding quasiequilibrium at the same point. 
The Ehrenfests' regularisation [6,7] provides "entropy trimming": we monitor local deviation of $f$ from the corresponding quasiequilibrium, and when $\Delta S(f)(\boldsymbol{x})$ exceeds a pre-specified threshold value $\delta$, perform local Ehrenfests' steps to the corresponding equilibrium: $f \mapsto f^{*}$ at those points.

So that the Ehrenfests' steps are not allowed to degrade the accuracy of LBGK it is pertinent to select the $k$ sites with highest $\Delta S>\delta$. The a posteriori estimations of added dissipation could easily be performed by analysis of entropy production in the Ehrenfests' steps. Numerical experiments show (see, e.g., Refs. [6,7]) that even a small number of such steps drastically improve stability.

To avoid the change of accuracy order "on average", the number of sites with this step should be $\leq \mathcal{O}(N h / L)$ where $N$ is the total number of sites, $h$ is the step of the space discretization and $L$ is the macroscopic characteristic length. But this rough estimate of accuracy on average might be destroyed by concentration of the Ehrenfests' steps in the most nonequilibrium areas, for example, in boundary layers. In that case, instead of the total number of sites $N$ in $\mathcal{O}(N h / L)$ we should take the number of sites in a specific region. The effects of concentration could be easily analysed a posteriori.

\subsection{Smooth limiters of nonequilibrium entropy}

The positivity rule and the Ehrenfests' regularisation provide rare, intense and localised corrections. Of course, it is easy and also computationally cheap to organise more gentle transformations with a smooth shift of highly nonequilibrium states to quasiequilibrium. The following regularisation transformation distributes its action smoothly:

$$
f \mapsto f^{*}+\phi(\Delta S(f))\left(f-f^{*}\right) .
$$

The choice of function $\phi$ is highly ambiguous; for example, $\phi=1 /\left(1+\alpha \Delta S^{k}\right)$ for some $\alpha>0$ and $k>0$. There are two significantly different choices: (i) ensemble-independent $\phi$ (i.e., the value of $\phi$ depends on local value of $\Delta S$ only) and (ii) ensemble-dependent $\phi$, for example

$$
\phi(\Delta S)=\frac{1+(\Delta S /(\alpha \mathrm{E}(\Delta S)))^{k-1 / 2}}{1+(\Delta S /(\alpha \mathrm{E}(\Delta S)))^{k}},
$$

where $\mathrm{E}(\Delta S)$ is the average value of $\Delta S$ in the computational area, $k \geq 1$, and $\alpha \gtrsim 1$. For small $\Delta S, \phi(\Delta S) \approx 1$ and for $\Delta S \gg \alpha \mathrm{E}(\Delta S)$ it tends to $\sqrt{\alpha \mathrm{E}(\Delta S) / \Delta S}$. It is easy to select an ensemble-dependent $\phi$ with control of total additional dissipation.

\subsection{Monitoring of total dissipation}

For given $\beta$, the entropy production in one LBGK step in quadratic approximation for $\Delta S$ is

$$
\delta_{\mathrm{LBGK}} S \approx\left[1-(2 \beta-1)^{2}\right] \sum_{x} \Delta S(\boldsymbol{x}),
$$

where $\boldsymbol{x}$ is the grid point, $\Delta S(\boldsymbol{x})$ is nonequilibrium entropy (9) at point $\boldsymbol{x}, \delta_{\mathrm{LBGK}} S$ is the total entropy production in a single LBGK step. It would be desirable if the total entropy production for the limiter $\delta_{\lim } S$ was small relative to $\delta_{\text {LBGK }} S$ :

$$
\delta_{\lim } S<\delta_{0} \delta_{\mathrm{LBGK}} S .
$$

A simple ensemble-dependent limiter (perhaps, the simplest one) for a given $\delta_{0}$ operates as follows. Let us collect the histogram of the $\Delta S(\boldsymbol{x})$ distribution, and estimate the distribution density, $p(\Delta S)$. We have to estimate a value $\Delta S_{0}$ that satisfies the following equation:

$$
\int_{\Delta S_{0}}^{\infty} p(\Delta S)\left(\Delta S-\Delta S_{0}\right) \mathrm{d} \Delta S=\delta_{0}\left[1-(2 \beta-1)^{2}\right] \int_{0}^{\infty} p(\Delta S) \Delta S \mathrm{~d} \Delta S .
$$

In order not to affect distributions with small expectation of $\Delta S$, we choose a threshold $\Delta S_{\mathrm{t}}=\max \left\{\Delta S_{0}, \delta\right\}$, where $\delta$ is some predefined value (as in the Ehrenfests' regularisation). For states at sites with $\Delta S \geq \Delta S_{\mathrm{t}}$ we provide homothety 
with equilibrium centre $f^{*}$ and coefficient $\sqrt{\Delta S_{\mathrm{t}} / \Delta S}$ (in quadratic approximation for nonequilibrium entropy):

$$
f(\boldsymbol{x}) \mapsto f^{*}(\boldsymbol{x})+\sqrt{\frac{\Delta S_{\mathrm{t}}}{\Delta S}}\left(f(\boldsymbol{x})-f^{*}(\boldsymbol{x})\right) .
$$

\subsection{Median entropy filter}

The limiters described above provide pointwise correction of nonequilibrium entropy at the "most nonequilibrium" points. Due to the pointwise nature, the technique does not introduce any nonisotropic effects, and provides some other benefits. But if we involve the local structure, we can correct local non-monotone irregularities without touching regular fragments. For example, we can discuss monotone increase or decrease of nonequilibrium entropy as regular fragments and concentrate our efforts on reduction of "speckle noise" or "salt and pepper noise". This approach allows us to use the accessible resource of entropy change (12) more thriftily. Salt and pepper noise is a form of noise typically observed in images. It represents itself as randomly occurring white (maximal brightness) and black pixels. For this kind of noise, conventional low pass filtering, e.g., mean filtering or Gaussian smoothing, is unsuccessful because the perturbed pixel value can vary significantly from both the original and the mean value. For this type of noise, median filtering is a common and effective noise reduction method. Median filtering is a common step in image processing [30] for the smoothing of signals and the suppression of impulse noise with preservation of edges.

The median is a more robust average than the mean (or the weighted mean) and so a single very unrepresentative value in a neighbourhood will not affect the median value significantly. Hence, we suppose that the median entropy filter will work better than entropy convolution filters.

For the nonequilibrium entropy field, the median filter considers each site in turn and looks at its nearby neighbours. It replaces the nonequilibrium entropy value $\Delta S$ at the point with the median of those values $\Delta S_{\text {med }}$, then updates $f$ by the transformation (14) with the homothety coefficient $\sqrt{\Delta S_{\text {med }} / \Delta S}$. The median, $\Delta S_{\text {med }}$, is calculated by first sorting all the values from the surrounding neighbourhood into numerical order and then replacing that being considered with the middle value. For example, if a point has 3 nearest neighbours including itself, then after sorting we have 3 values $\Delta S: \Delta S_{1} \leq \Delta S_{2} \leq \Delta S_{3}$. The median value is $\Delta S_{\text {med }}=\Delta S_{2}$. For 9 nearest neighbours (including itself) we have after sorting $\Delta S_{\text {med }}=\Delta S_{5}$. For 27 nearest neighbours $\Delta S_{\text {med }}=\Delta S_{14}$.

We accept only dissipative corrections (those resulting in a decrease of $\Delta S, \Delta S_{\text {med }}<\Delta S$ ) because of the second law of thermodynamics. The analogue of (13) is also useful for acceptance of the most significant corrections. In "salt and pepper" terms, we correct the salt (where $\Delta S$ exceeds the median value) and do not touch the pepper.

\subsection{Monotonic and double-monotonic limiters}

Two monotonicity properties are important in the theory of nonequilibrium entropy limiters:

(1) a limiter should move the distribution to equilibrium: in all cases of (1) $0 \leq \phi \leq 1$; this is the dissipativity condition which means that limiters never produce negative entropy;

(2) a limiter should not change the order of states on the line: if for two distributions with the same moments, $f$ and $f^{\prime}, f^{\prime}-f^{*}=x\left(f-f^{*}\right)$ and $\Delta S(f)>\Delta S\left(f^{\prime}\right)$ before the limiter transformation, then the same inequality should hold after the limiter transformation too; for example, for the limiter (10) it means that $\Delta S\left(f^{*}+x \phi\left(\Delta S\left(f^{*}+x\left(f-f^{*}\right)\right)\right)\left(f-f^{*}\right)\right)$ is a monotonically increasing function of $x>0$.

In quadratic approximation,

$$
\begin{aligned}
& \Delta S\left(f^{*}+x\left(f-f^{*}\right)\right)=x^{2} \Delta S(f), \\
& \Delta S\left(f^{*}+x \phi\left(\Delta S\left(f^{*}+x\left(f-f^{*}\right)\right)\right)\left(f-f^{*}\right)\right)=x^{2} \phi^{2}\left(x^{2} \Delta S(f)\right),
\end{aligned}
$$

and the second monotonicity condition transforms into the following requirement: $y \phi\left(y^{2} s\right)$ is a monotonically increasing (not decreasing) function of $y>0$ for any $s>0$.

If a limiter satisfies both monotonicity conditions, we call it "double monotonic". For example, the Ehrenfests' regularisation satisfies the first monotonicity condition, but violates the second one. The limiter (11) violates the first condition for small $\Delta S$, but is dissipative and satisfies the second one in quadratic approximation for large $\Delta S$. The limiter with $\phi=1 /\left(1+\alpha \Delta S^{k}\right)$ always satisfies the first monotonicity condition, violates the second if $k>1 / 2$, and 
is double monotonic (in quadratic approximation for the second condition) if $0<k \leq 1 / 2$. The threshold limiter (14) is also double monotonic.

For smooth functions, the condition of double monotonicity (in quadratic approximation) is equivalent to the system of differential inequalities

$$
\begin{aligned}
& \phi(x)+2 x \phi^{\prime}(x) \geq 0 ; \\
& \phi^{\prime}(x) \leq 0 .
\end{aligned}
$$

The initial condition $\phi(0)=1$ means that in the limit $\Delta S \rightarrow 0$ limiters do not affect the flow. Following these inequalities we can write: $2 x \phi^{\prime}(x)=-\eta(x) \phi(x)$, where $0 \leq \eta(x) \leq 1$. The solution of these inequalities with the initial condition is

$$
\phi(x)=\exp \left(-\frac{1}{2} \int_{0}^{x} \frac{\eta(\chi)}{\chi} \mathrm{d} \chi\right),
$$

if the integral on the right-hand side exists. This is a general solution for double-monotonic limiters (in the second approximation for entropy). If $\eta(x)$ is the Heaviside step function, $\eta(x)=H\left(x-\Delta S_{\mathrm{t}}\right)$ with threshold value $\Delta S_{\mathrm{t}}$, then the general solution (15) gives us the threshold limiter. If, for example, $\eta(x)=x^{k} /\left(\Delta S_{\mathrm{t}}^{k}+x^{k}\right)$, then

$$
\phi(x)=\left(1+\frac{x^{k}}{\Delta S_{\mathrm{t}}^{k}}\right)^{-\frac{1}{2 k}} .
$$

This special form of limiter function is attractive because for small $x$ it gives

$$
\phi(x)=1-\frac{1}{2 k} \frac{x^{k}}{\Delta S_{\mathrm{t}}^{k}}+o\left(x^{k}\right) .
$$

Thus, the limiter does not affect the motion up to the $(k+1)$ st order, and the macroscopic equations coincide with the macroscopic equations for LBM without limiters up to the $(k+1)$ st order in powers of deviation from quasiequilibrium. Furthermore, for large $x$ we get the $k$ th-order approximation to the threshold limiter (14):

$$
\phi(x)=\sqrt{\frac{\Delta S_{\mathrm{t}}}{x}}+o\left(x^{-k}\right) .
$$

Of course, it is not forbidden to use any type of limiter under the local and global control of dissipation, but double-monotonic limiters provide some natural properties automatically, without additional care.

\section{Numerical experiments}

To conclude this paper we report some numerical experiments conducted to demonstrate the performance of some of the proposed nonequilibrium entropy limiters for LBM from Section 3.

\subsection{Velocities and equilibria}

We will perform simulations using both entropic and non-entropic local equilibria, but we always work with an athermal LBM model. Whenever we use non-entropic equilibria we employ Kullback entropy (7).

In $1 \mathrm{D}$, we use a lattice with spacing and time step $\delta t=1$ and a discrete velocity set $\left\{v_{1}, v_{2}, v_{3}\right\}:=\{0,-1,1\}$ so that the model consists of static, left-moving and right-moving populations only. The subscript $i$ denotes population (not lattice site number) and $f_{1}, f_{2}$ and $f_{3}$ denote the static, left-moving and right-moving populations, respectively. The entropy is $S=-H$, with

$$
H=f_{1} \log \left(f_{1} / 4\right)+f_{2} \log \left(f_{2}\right)+f_{3} \log \left(f_{3}\right)
$$


(see, e.g., Ref. [23]) and, for this entropy, the local entropic equilibrium state $f^{*}$ is available explicitly:

$$
\begin{aligned}
f_{1}^{*} & =\frac{2 \rho}{3}\left(2-\sqrt{1+3 u^{2}}\right) \\
f_{2}^{*} & =\frac{\rho}{6}\left((3 u-1)+2 \sqrt{1+3 u^{2}}\right), \\
f_{3}^{*} & =-\frac{\rho}{6}\left((3 u+1)-2 \sqrt{1+3 u^{2}}\right),
\end{aligned}
$$

where

$$
\rho:=\sum_{i} f_{i}, \quad u:=\frac{1}{\rho} \sum_{i} v_{i} f_{i} .
$$

The standard non-entropic polynomial equilibria [33] are

$$
\begin{aligned}
f_{1}^{*} & =\frac{2 \rho}{3}\left(1-\frac{3 u^{2}}{2}\right), \\
f_{2}^{*} & =\frac{\rho}{6}\left(1-3 u+3 u^{2}\right), \\
f_{3}^{*} & =\frac{\rho}{6}\left(1+3 u+3 u^{2}\right) .
\end{aligned}
$$

In 2D, we employ a uniform nine-speed square lattice with discrete velocities $\left\{\boldsymbol{v}_{i} \mid i=0,1, \ldots, 8\right\}: \boldsymbol{v}_{0}=0$, $v_{i}=(\cos ((i-1) \pi / 2), \sin ((i-1) \pi / 2))$ for $i=1,2,3,4, v_{i}=\sqrt{2}\left(\cos \left((i-5) \frac{\pi}{2}+\frac{\pi}{4}\right), \sin \left((i-5) \frac{\pi}{2}+\frac{\pi}{4}\right)\right)$ for $i=5,6,7,8$. The numbering $f_{0}, f_{1}, \ldots, c, f_{8}$ are for the static, east, north, west, south, northeast, northwest, southwest and southeast-moving populations, respectively. As usual, the entropic equilibrium state, $f^{*}$, can be uniquely determined by maximising an entropy functional

$$
S(f)=-\sum_{i} f_{i} \log \left(\frac{f_{i}}{W_{i}}\right),
$$

subject to the constraints of conservation of mass and momentum [2]:

$$
f_{i}^{*}=\rho W_{i} \prod_{j=1}^{2}\left(2-\sqrt{1+3 u_{j}^{2}}\right)\left(\frac{2 u_{j}+\sqrt{1+3 u_{j}^{2}}}{1-u_{j}}\right)^{v_{i, j}} .
$$

Here, the lattice weights, $W_{i}$, are given lattice-specific constants: $W_{0}=4 / 9, W_{1,2,3,4}=1 / 9$ and $W_{5,6,7,8}=1 / 36$. Analogously to (19), the macroscopic variables $\rho$ and $\boldsymbol{u}=\left(u_{1}, u_{2}\right)$ are the zeroth and first moments of the distribution $f$, respectively. The standard non-entropic polynomial equilibria [33] are

$$
f_{i}^{*}=\rho W_{i}\left(1+3 v_{i} \boldsymbol{u}+\frac{9\left(v_{i} \boldsymbol{u}\right)^{2}}{2}-\frac{3 \boldsymbol{u}^{2}}{2}\right) .
$$

\section{2. $L B G K$ and $E L B M$}

The governing equations for LBGK are

$$
f_{i}\left(x+v_{i}, t+1\right)=f_{i}^{*}(x, t)+(2 \beta-1)\left(f_{i}^{*}(x, t)-f_{i}(x, t)\right),
$$

where $\beta=1 /(2 v+1)$.

For ELBM (4) the governing equations are

$$
f_{i}\left(x+v_{i}, t+1\right)=(1-\beta) f_{i}^{*}(x, t)+\beta \tilde{f}_{i}(x, t),
$$



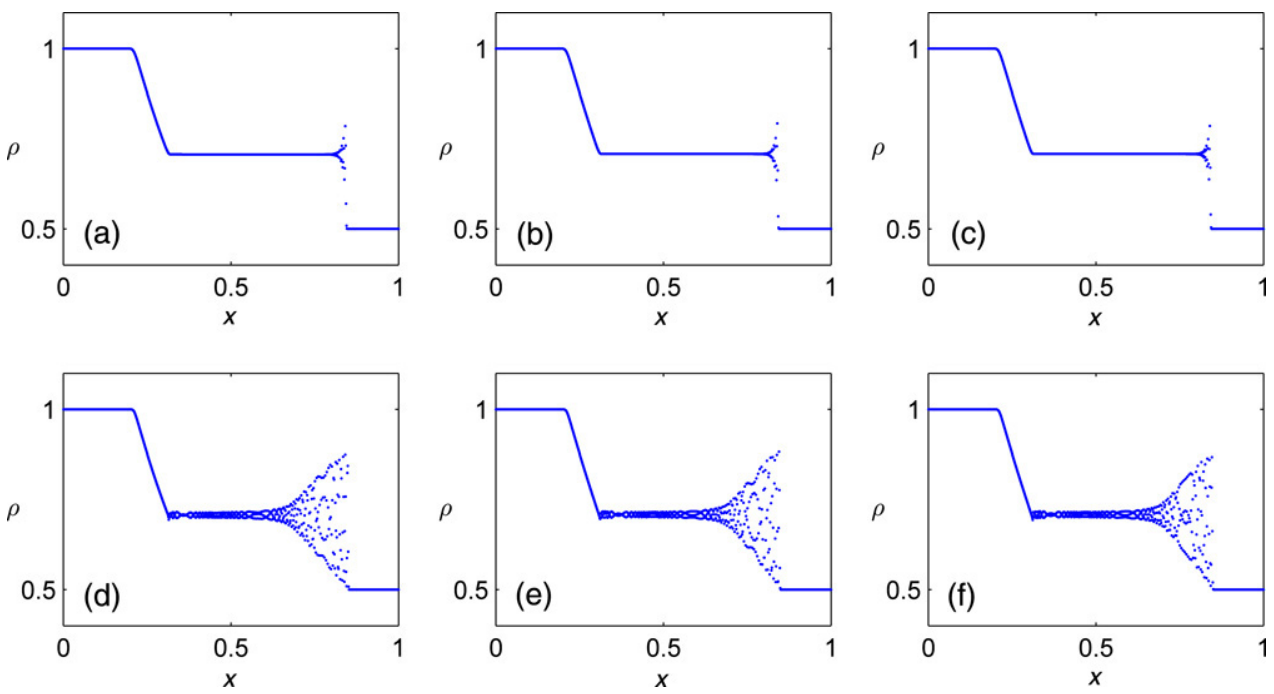

Fig. 2. Density profile of the 1:2 isothermal shock tube simulation after 400 time steps using (a) LBGK (23) with polynomial quasiequilibria (20) $\left[v=3.3333 \times 10^{-2}\right]$; (b) LBGK (23) with entropic quasiequilibria (18) $\left[v=3.3333 \times 10^{-2}\right]$; (c) ELBM $(24)\left[v=3.3333 \times 10^{-2}\right]$; (d) LBGK $(23)$ with polynomial quasiequilibria (20) $\left[v=10^{-9}\right]$; (e) LBGK (23) with entropic quasiequilibria $(18)\left[v=10^{-9}\right]$; (f) ELBM (24) $\left[v=10^{-9}\right]$.

with $\beta$ as above and $\tilde{f}=(1-\alpha) f+\alpha f^{*}$. The parameter, $\alpha$, is chosen to satisfy a constant entropy condition. This involves finding the nontrivial root of the equation

$$
S\left((1-\alpha) f+\alpha f^{*}\right)=S(f) .
$$

To solve (25) numerically we employ a robust routine based on bisection. The root is solved to an accuracy of $10^{-15}$ and we always ensure that the returned value of $\alpha$ does not lead to a numerical entropy decrease. We stipulate that if, at some site, no nontrivial root of (25) exists, we will employ the positivity rule instead (Fig. 1).

\subsection{Shock tube}

The 1D shock tube for a compressible athermal fluid is a standard benchmark test for hydrodynamic codes. Our computational domain will be the interval $[0,1]$ and we discretize this interval with 801 uniformly spaced lattice sites. We choose the initial density ratio as $1: 2$ so that for $x \leq 400$ we set $\rho=1.0$; otherwise we set $\rho=0.5$.

In the first test we present three possible combinations of two choices of equilibria, polynomial (20) or entropic (18), and two choices of stepping, LBGK (23) or ELBM (24). We solve (25) to an accuracy of $10^{-15}$. The results, which are self-explanatory, are presented in Fig. 2 for the kinematic viscosity $v=3.3333 \times 10^{-2}$ and $v=10^{-9}$ in dimensionless units.

For shock tube experiments, the introduction of local dissipation in thin zones near shocks has a similar effect to the introduction of global dissipation in the whole domain. Even the introduction of local dissipation at one point suppresses spurious oscillations [6,7]. In the absence of any dissipation mechanism, dispersive oscillations on the mesh scale have to appear in shock regions [26].

It is necessary to mention that for the difference schemes without artificial numerical dissipation studied in Ref. [36], spurious oscillations in the immediate neighbourhoods of shocks could be suppressed by sufficiently high viscosity or heat conduction only (and the roles of viscosity and heat conduction in forming sharp monotone profiles are different). No limiters were added in Ref. [36], but instead, the viscous and heat conduction terms in the Navier-Stokes equations are found to serve as accurate edge detectors.

Following the idea of comparison of the action of limiters with physical dissipation, we present LBGK computations of shocks without any limiter as a reference point for testing limiters (Fig. 3).

For all further shock tube experiments with limiters we will fix the kinematic viscosity of the fluid at $v=10^{-9}$ (the "bulk viscosity") in dimensionless units. Of course, by using limiters an additional viscosity is produced in thin zones with high gradients, and this $v=10^{-9}$ characterises the LBM gas before the introduction of limiters. Additional dissipation is discussed in Section 3.4 and below in Section 5. 

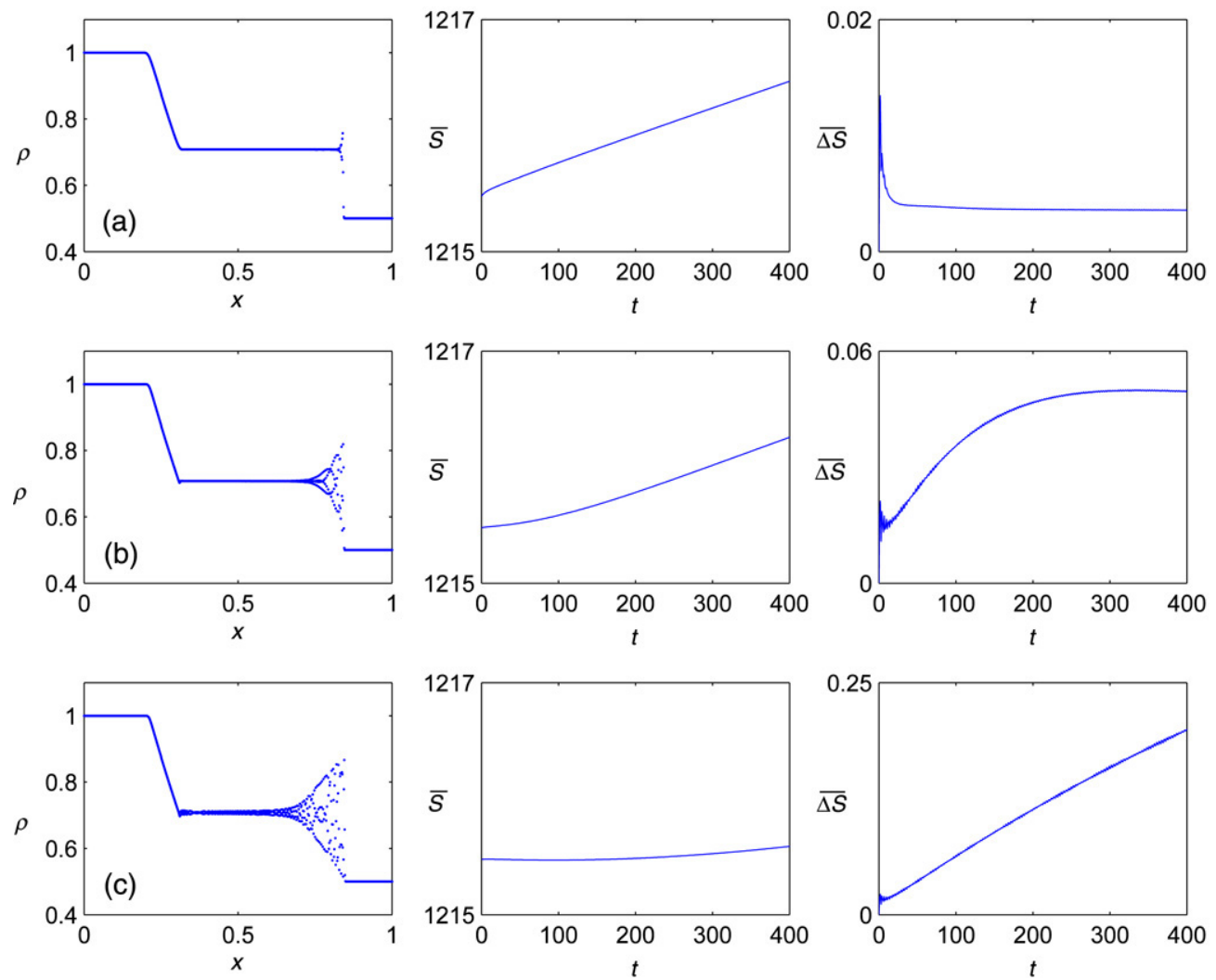

Fig. 3. Density profile of the 1:2 athermal shock tube simulation with (a) $v=0.066$, (b) $v=0.0066$ and (c) $v=0.00066$ after 400 time steps using LBGK (23) without any limiter. Total entropy and nonequilibrium entropy time histories are displayed in the adjacent panels. Entropic equilibria (18) with perfect entropy (17) are used.

Now, we would like to demonstrate just a representative sample of the many possibilities of limiters suggested in Section 3. In each case the limiter is implemented by a post-processing routine immediately following the collision step (either LBGK (23) or ELBM (24)). Here, we will only consider LBGK collisions.

The post-processing step adjusts $f$ using the update formula

$$
f \mapsto f^{*}+\phi(\Delta S)\left(f-f^{*}\right),
$$

where $\Delta S$ is defined by (9) and $\phi$ is a limiter function.

For the Ehrenfests' regularisation one would choose

$$
\phi(\Delta S)(x)= \begin{cases}1, & \Delta S(x) \leq \delta \\ 0, & \text { otherwise }\end{cases}
$$

where $\delta$ is a pre-specified threshold value. Furthermore, it is pertinent to select just $k$ sites with highest $\Delta S>\delta$. This limiter has been previously applied to the shock tube problem in Refs. [6-8] and we will not reproduce those results here.

Instead, our first example will be the following smooth limiter:

$$
\phi(\Delta S)=\frac{1}{1+\alpha \Delta S^{k}} .
$$

This limiter (26) always satisfies the condition $\phi(0)=1$, and $\phi^{\prime}(0)=0$ for $k>1$. For this limiter, we will fix $k=1 / 2$ (so that the limiter is double monotonic in quadratic approximation to entropy) and compare the density profiles for $\alpha=\delta /\left(\mathrm{E}(\Delta S)^{k}\right), \delta=0.1,0.01,0.001$ (Fig. 4). We have also ensured an ensemble-dependent limiter because of the 

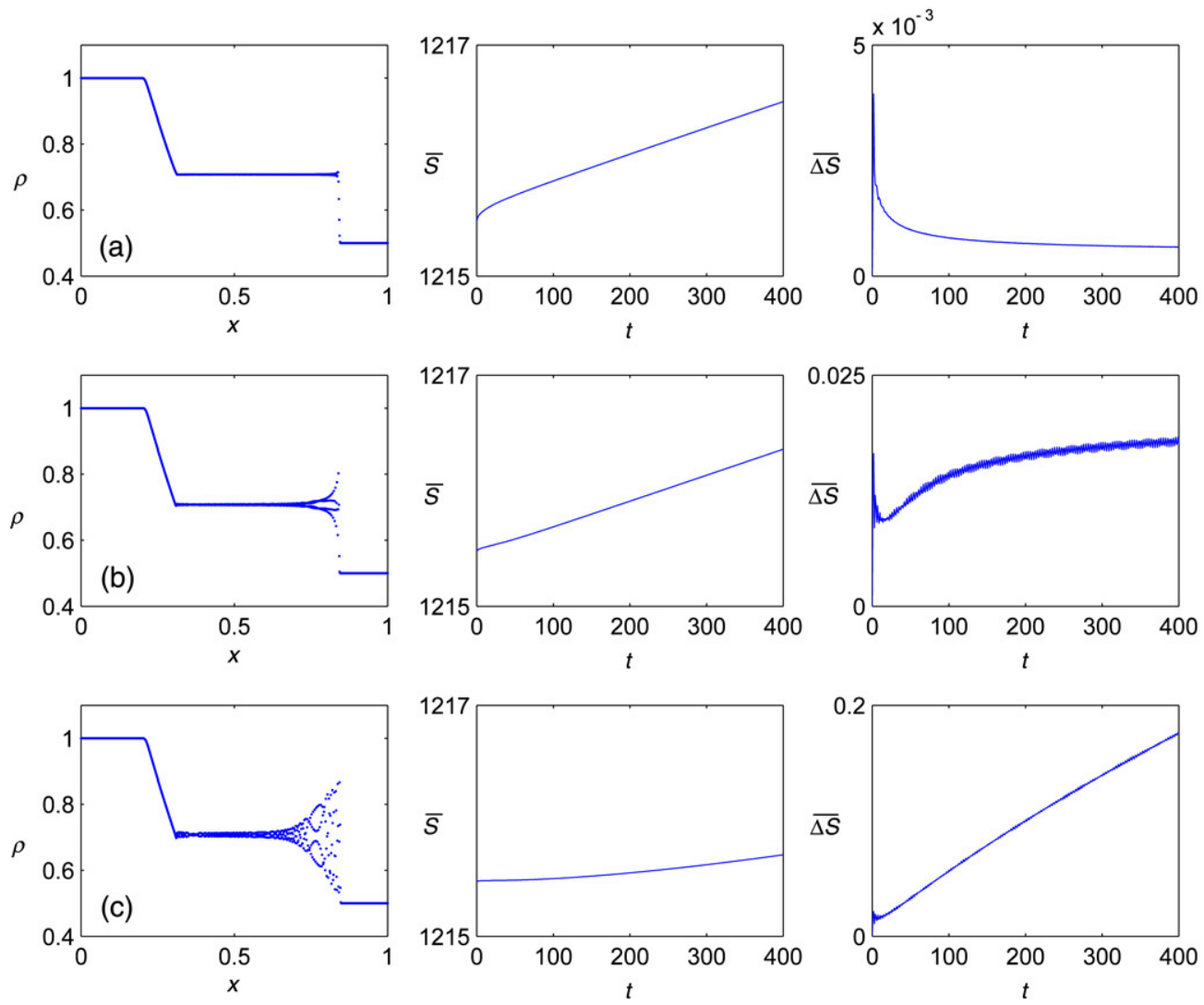

Fig. 4. Density profile of the 1:2 athermal shock tube simulation with $v=10^{-9}$ after 400 time steps using LBGK (23) and the smooth limiter (26) with $k=1 / 2, \alpha=\delta /\left(\mathrm{E}(\Delta S)^{k}\right)$ and (a) $\delta=0.1$; (b) $\delta=0.01$ and (c) $\delta=0.001$. Total entropy and nonequilibrium entropy time histories for each parameter set $\{k, \alpha(\delta)\}$ are displayed in the adjacent panels. The last case (c) is close to LBGK simulation without limiters.

dependence of $\alpha$ on the average $\mathrm{E}(\Delta S)$. We accompany each panel with the total entropy and nonequilibrium entropy histories. Note the different scales for nonequilibrium entropy. Note also that entropy (necessarily) now grows due to the additional dissipation.

The second example is the smooth limiter (26) with $k=1$ (Fig. 5). It is worthwhile to mention that for the same parameter $\delta$ this limiter suppresses spurious oscillations more effectively. For example, for $\delta=0.001$, for $k=1 / 2$ we see almost the same post-shock oscillations as for the system without limiter, whereas the same picture for $k=1$ already differs. For points with $\Delta S<\mathrm{E}(\Delta S)$ the limiter with $k=1$ and the same $\delta$ affects the flow less than the limiter with $k=1 / 2$, whereas for $\Delta S>\mathrm{E}(\Delta S)$ it works more intensively. We can guess that for effective suppression of post-shock oscillations it is important how the limiter behaves for $\Delta S \gg \mathrm{E}(\Delta S)$. More detailed discussion is presented in Section 5. We should mention that this limiter is not double monotonic, but this caused no problems in this specific experiment.

Our next example (Fig. 6) considers the threshold filter (13). In this example we choose the estimates $\Delta S_{0}=$ $5 \mathrm{E}(\Delta S), 10 \mathrm{E}(\Delta S), 20 \mathrm{E}(\Delta S)$ and fix the tolerance $\delta=0$ so that the influence of the threshold alone can be studied. Only entropic adjustments are accepted in the limiter: $\Delta S_{t} \leq \Delta S$. As the threshold increases, nonequilibrium entropy grows faster and spurious oscillations begin to appear.

Finally, we test the median filter (Fig. 7). We choose a minimal filter so that only the nearest neighbours are considered. As with the threshold filter, we introduce a tolerance $\delta$ and we try the values $\delta=10^{-3}, 10^{-4}, 10^{-5}$. Only entropic adjustments are accepted in the limiter: $\Delta S_{\text {med }} \leq \Delta S$.

We have seen that each of the examples we have considered (Figs. 4-7) is capable of subduing spurious post-shock oscillations compared with LBGK. Of course, on limiting nonequilibrium entropy the result is necessarily an increase in entropy. 

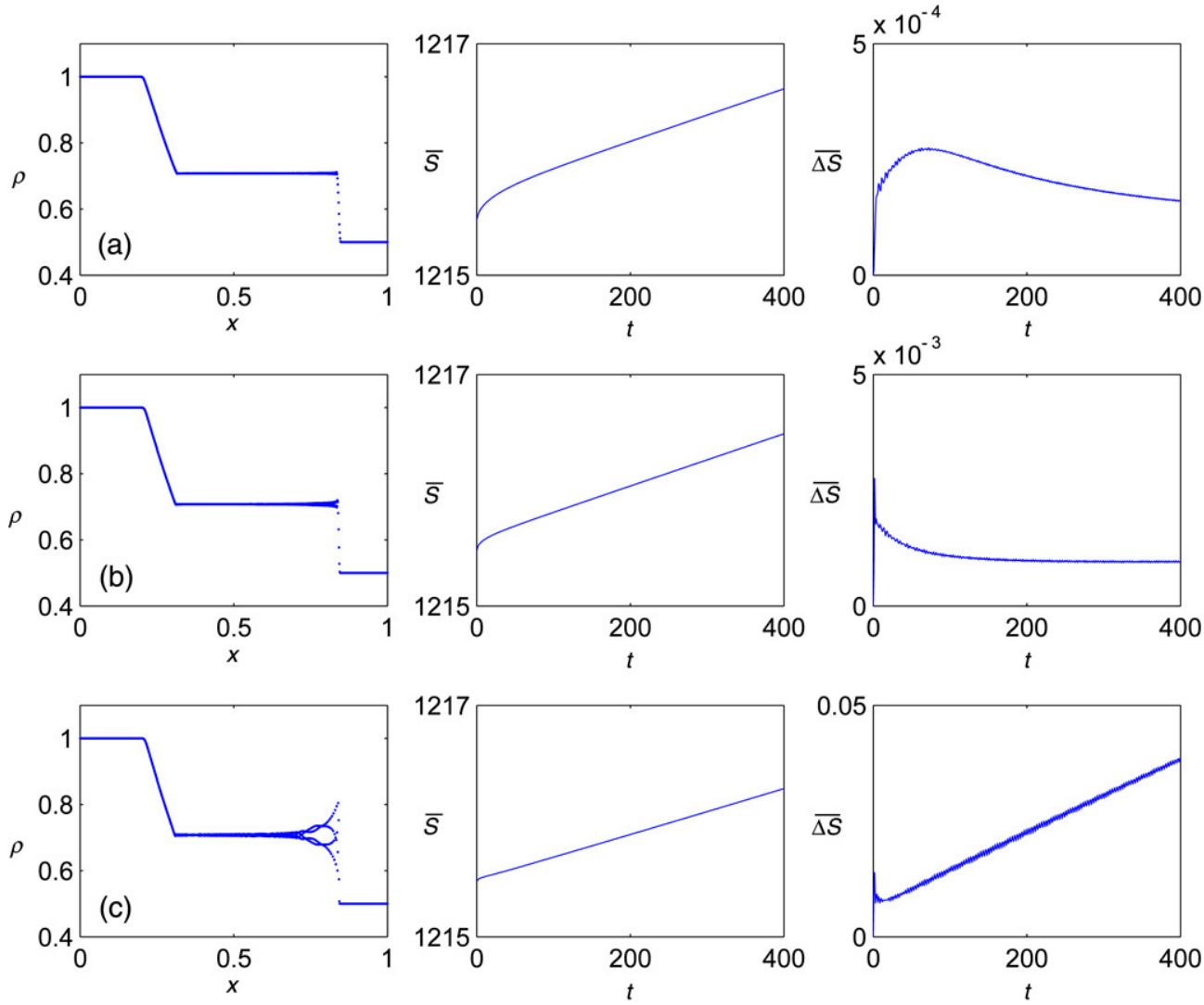

Fig. 5. Density profile of the 1:2 athermal shock tube simulation with $v=10^{-9}$ after 400 time steps using LBGK (23) and the smooth limiter (26) with $k=1, \alpha=\delta /\left(\mathrm{E}(\Delta S)^{k}\right)$ and (a) $\delta=0.1$; (b) $\delta=0.01$ and (c) $\delta=0.001$. Total entropy and nonequilibrium entropy time histories for each parameter set $\{k, \alpha(\delta)\}$ are displayed in the adjacent panels.

From our experiences our recommendation is that the median filter is the superior choice amongst all the limiters suggested in Section 3. The action of the median filter is found to be both extremely gentle and, at the same time, very effective.

\subsection{Lid-driven cavity}

Our second numerical example is the classical 2D lid-driven cavity flow. A square cavity of side length $L$ is filled with fluid with kinematic viscosity $v$ (initially at rest) and driven by the cavity lid moving at a constant velocity $\left(u_{0}, 0\right)$ (from left to right in our geometry).

We will simulate the flow on a $100 \times 100$ grid using LBGK regularised with the median filter limiter. Unless otherwise stated, we use entropic equilibria (21). The implementation of the filter is as follows: the filter is not applied to boundary nodes; for nodes which immediately neighbour the boundary the stencil consists of the three nearest neighbours (including itself) closest to the boundary; for all other nodes the minimal stencil of nine nearest neighbours is used.

We have purposefully selected such a coarse grid simulation because it is readily found that, on this problem, unregularised LGBK fails (blows up) for all but the most modest Reynolds numbers $R e:=L u_{0} / v$.

\subsubsection{Steady state vortex centres}

For modest Reynolds number the system settles to a steady state in which the dominant features are a primary central rotating vortex, with several counter-rotating secondary vortices located in the bottom left, bottom right (and possibly top left) corners. 

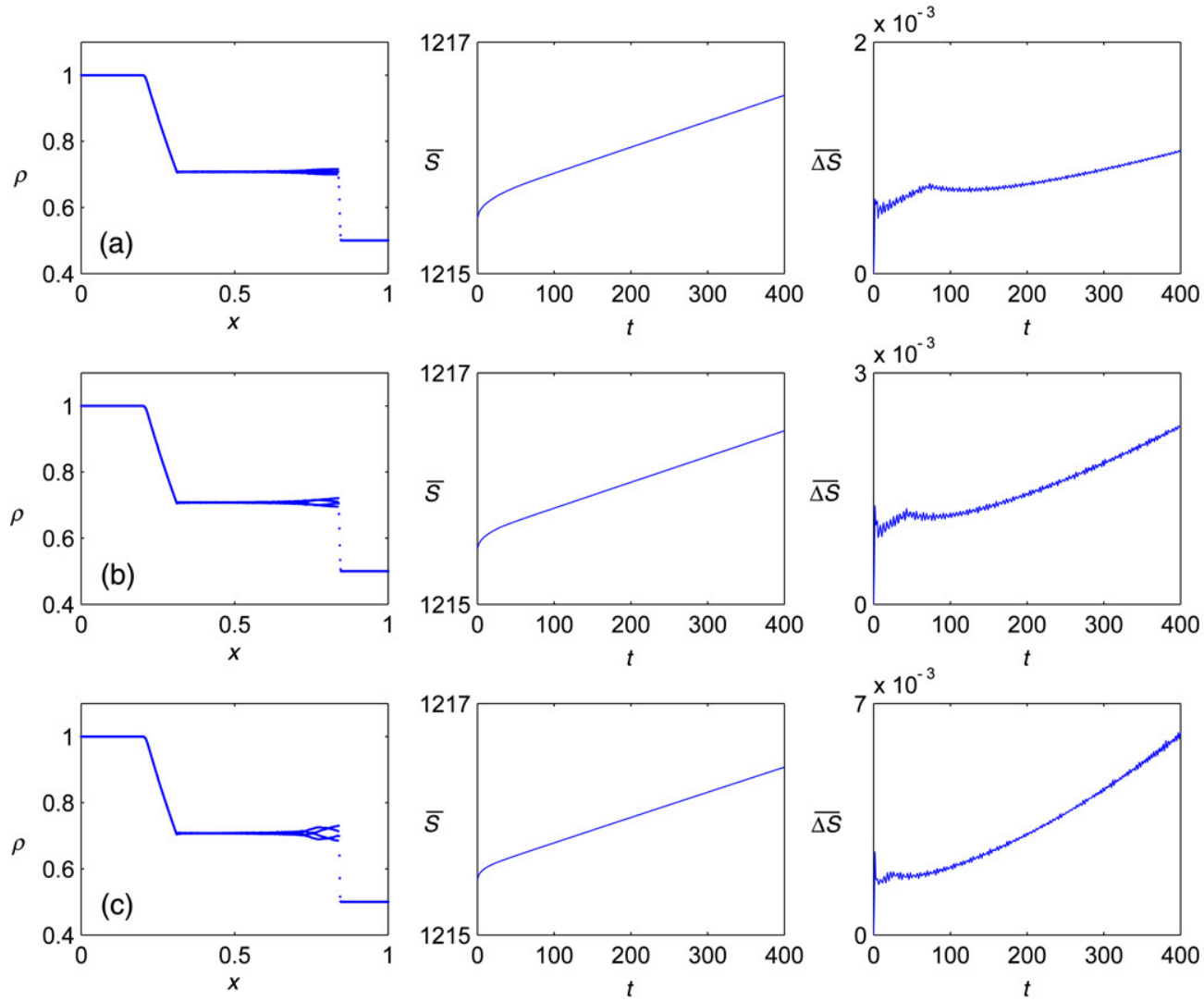

Fig. 6. Density profile of the 1:2 athermal shock tube simulation with $v=10^{-9}$ after 400 time steps using LBGK (23) and the threshold limiter (13) with (a) $\Delta S_{\mathrm{t}}=5 \mathrm{E}(\Delta S)$; (b) $\Delta S_{\mathrm{t}}=10 \mathrm{E}(\Delta S)$ and (c) $\Delta S_{\mathrm{t}}=20 \mathrm{E}(\Delta S)$. Total entropy and nonequilibrium entropy time histories for each threshold $\Delta S_{\mathrm{t}}$ are displayed in the adjacent panels.

The steady state has been extensively investigated in the literature. The study of Hou et al. [21] simulates the flow over a range of Reynolds numbers using unregularised LBGK on a $256 \times 256$ grid. Primary and secondary vortex centre data are provided. We compare this same statistic for the present median filtered coarse grid simulation. We will employ the same convergence criteria as are used in Ref. [21]. Namely, we deem that steady state has been reached by ensuring that the difference between the maximum value of the stream function for 10,000 successive time steps is less than $10^{-5}$. The stream function, which is not a primary variable in the LBM simulation, is obtained from the velocity data by integration using Simpson's rule. Vortex centres are characterised as local extrema of the stream function.

We compare our results with the LBGK simulations in Refs. [21] and [37]. To align ourselves with these studies we specify the following boundary condition: lid profile is constant; remaining cavity walls are subject to the "bounceback" condition [33]. In our simulations, the initial uniform fluid density profile is $\rho=2.7$ and the velocity of the lid is $u_{0}=1 / 10$ (in lattice units).

Collected in Table 1, for $R e=2000,5000$ and 7500, are the coordinates of the primary and secondary vortex centres using (a) unregularised LBGK; (b) LBGK with median filter limiter $\left(\delta=10^{-3}\right)$; (c) LBGK with median filter limiter $\left(\delta=10^{-4}\right)$, all with non-entropic polynomial equilibria (22). Lines (d), (e) and (f) are the same but with entropic equilibria (21). The remaining lines of Table 1 are as follows: (g) literature data [21] (unregularised LBGK on a $256 \times 256$ grid); (h) literature data [37] (positivity rule); (i) literature data [37] (ELBM). With the exception of $(\mathrm{g})$, all simulations are conducted on a $100 \times 100$ grid. The top left vortex does not appear at $R e=2000$ and no data were provided for it in [37] at $R e=5000$. The unregularised LBGK $R e=7500$ simulation blows up in finite time and the simulation becomes meaningless. The $y$-coordinates of the two lower vortices at $R e=5000$ in (i) appear anomalously small and were not reproduced by our experiments with the positivity rule (not shown). 

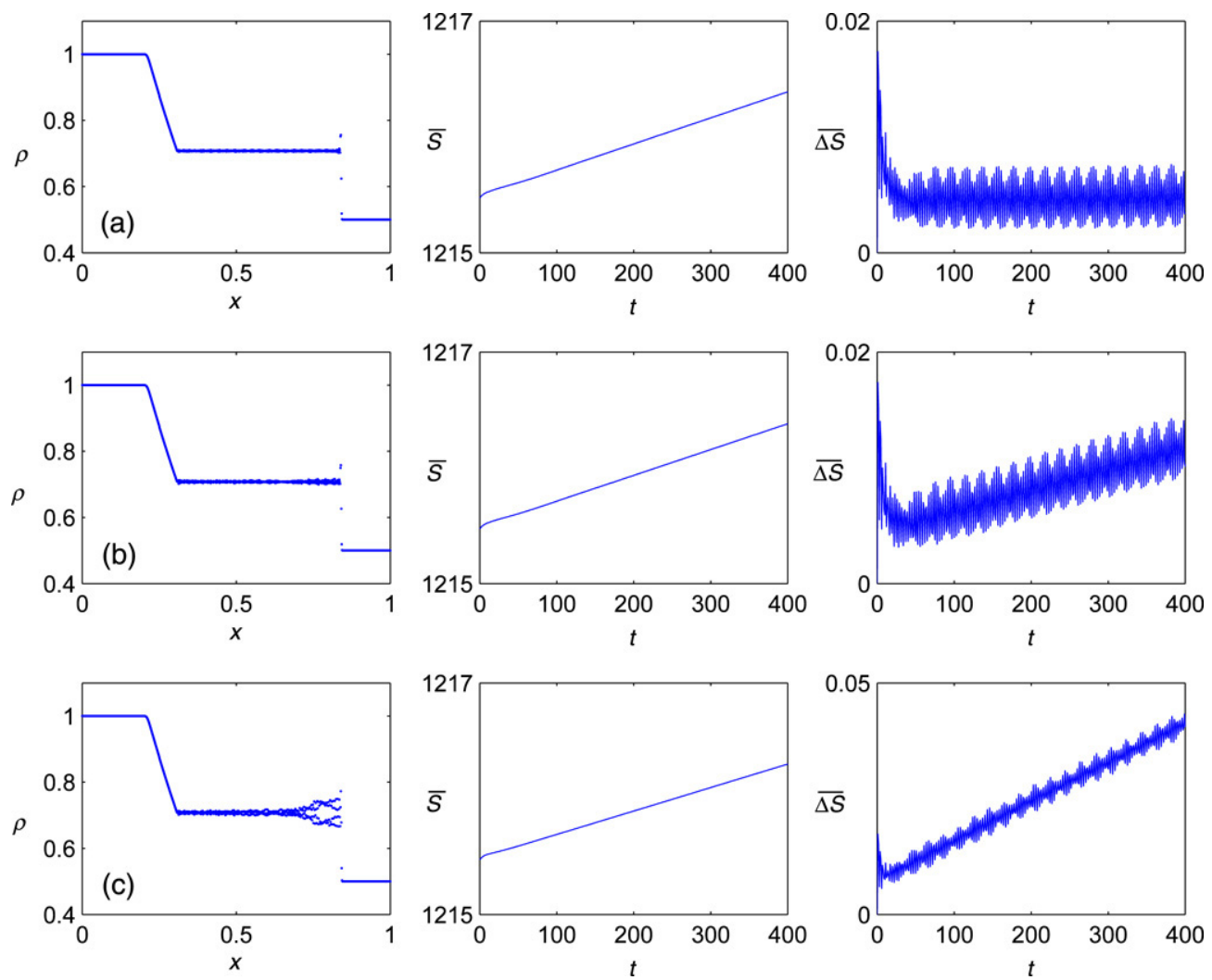

Fig. 7. Density profile of the $1: 2$ athermal shock tube simulation with $v=10^{-9}$ after 400 time steps using LBGK (23) and the minimal median limiter with (a) $\delta=10^{-5}$; (b) $\delta=10^{-4}$ and (c) $\delta=10^{-3}$. Total entropy and nonequilibrium entropy time histories for each tolerance $\delta$ are displayed in the adjacent panels.

We have conducted two runs of the experiment with the median filter parameter $\delta=10^{-3}$ and $\delta=10^{-4}$. Despite the increased number of realisations the vortex centre locations remain effectively unchanged and we detect no significant variation between the two runs. This demonstrates the gentle nature of the median filter. At Reynolds number $R e=2000$ the median filter has no effect at all on the vortex centres compared with LBGK.

We find no significant differences between the experiments with entropic and non-entropic polynomial equilibria in this test.

The coordinates of the primary vortex centre for unregularised LBGK at $R e=5000$ are already quite inaccurate as LBGK begins to lose stability. Stability is lost entirely at some critical Reynolds number $5000<R e \leq 7500$ and the simulation blows up.

Furthermore, we have agreement (within grid resolution) with the data given in Ref. [21]. Also compiled in Table 1 are the data from the limiter experiments conducted in Ref. [37] (although not explicitly discussed in the language of limiters by the authors of that work). In Ref. [37] the authors give vortex centre data for the positivity rule (Fig. 1) and for ELBM. In Ref. [37] the positivity rule is called FIX-UP.

As Reynolds number increases the flow in the cavity becomes no longer steady and a more complicated flow pattern emerges. On the way to a fully developed turbulent flow, the lid-driven cavity flow is known to undergo a series of period-doubling Hopf bifurcations. On our coarse grid, we observe that the coordinate of the primary vortex centre (maximum of the stream function) is a very robust feature of the flow, with little change between coordinates (no change in $y$-coordinates) computed at $R e=5000$ and $R e=7500$ with the median filter. On one hand, because of this observation it becomes inconclusive whether the median limiter is adding too much additional dissipation. On the other hand, a more studious choice of control criteria may indicate that the first bifurcation has already occurred by $R e=7500$. 
Table 1

Primary and secondary vortex centre coordinates for the lid-driven cavity flow at $R e=2000,5000,7500$

\begin{tabular}{|c|c|c|c|c|c|c|c|c|c|}
\hline \multirow[t]{2}{*}{$R e$} & & \multicolumn{2}{|l|}{ Primary } & \multicolumn{2}{|c|}{$\underline{\text { Lower left }}$} & \multicolumn{2}{|c|}{ Lower right } & \multicolumn{2}{|l|}{ Top left } \\
\hline & & $x$ & $y$ & $x$ & $y$ & $x$ & $y$ & $x$ & $y$ \\
\hline 2000 & (a) & 0.5253 & 0.5455 & 0.0909 & 0.1010 & 0.8384 & 0.1010 & Not applicable & \\
\hline 2000 & (b) & 0.5253 & 0.5455 & 0.0909 & 0.1010 & 0.8384 & 0.1010 & Not applicable & \\
\hline 2000 & (c) & 0.5253 & 0.5455 & 0.0909 & 0.1010 & 0.8384 & 0.1010 & Not applicable & \\
\hline 2000 & (d) & 0.5253 & 0.5455 & 0.0909 & 0.1010 & 0.8384 & 0.1010 & Not applicable & \\
\hline 2000 & (e) & 0.5253 & 0.5455 & 0.0909 & 0.1010 & 0.8384 & 0.1010 & Not applicable & \\
\hline 2000 & (f) & 0.5253 & 0.5455 & 0.0909 & 0.1010 & 0.8384 & 0.1010 & Not applicable & \\
\hline 2000 & (g) & 0.5255 & 0.5490 & 0.0902 & 0.1059 & 0.8471 & 0.0980 & Not applicable & \\
\hline 2000 & (h) & 0.5200 & 0.5450 & 0.0900 & 0.1000 & 0.8300 & 0.0950 & Not applicable & \\
\hline 2000 & (i) & 0.5200 & 0.5500 & 0.0890 & 0.1000 & 0.8300 & 0.1000 & Not applicable & \\
\hline 5000 & (a) & 0.5152 & 0.6061 & 0.0808 & 0.1313 & 0.7980 & 0.0707 & 0.0505 & 0.8990 \\
\hline 5000 & (b) & 0.5152 & 0.5354 & 0.0808 & 0.1313 & 0.8081 & 0.0808 & 0.0606 & 0.8990 \\
\hline 5000 & (c) & 0.5152 & 0.5354 & 0.0808 & 0.1313 & 0.8081 & 0.0808 & 0.0707 & 0.8889 \\
\hline 5000 & (d) & 0.5152 & 0.5960 & 0.0808 & 0.1313 & 0.8081 & 0.0808 & 0.0505 & 0.8990 \\
\hline 5000 & (e) & 0.5152 & 0.5354 & 0.0808 & 0.1313 & 0.8081 & 0.0808 & 0.0606 & 0.8990 \\
\hline 5000 & (f) & 0.5152 & 0.5354 & 0.0808 & 0.1313 & 0.8081 & 0.0808 & 0.0707 & 0.8889 \\
\hline 5000 & (g) & 0.5176 & 0.5373 & 0.0784 & 0.1373 & 0.8078 & 0.0745 & 0.0667 & 0.9059 \\
\hline 5000 & (h) & 0.5150 & 0.5680 & 0.0950 & 0.0100 & 0.8450 & 0.0100 & Not available & \\
\hline 5000 & (i) & 0.5150 & 0.5400 & 0.0780 & 0.1350 & 0.8050 & 0.0750 & Not available & \\
\hline 7500 & (a) & - & - & - & - & - & - & - & - \\
\hline 7500 & (b) & 0.5051 & 0.5354 & 0.0707 & 0.1515 & 0.7879 & 0.0707 & 0.0606 & 0.8990 \\
\hline 7500 & (c) & 0.5051 & 0.5354 & 0.0707 & 0.1515 & 0.7879 & 0.0707 & 0.0707 & 0.8889 \\
\hline 7500 & (d) & - & - & - & - & - & - & - & - \\
\hline 7500 & (e) & 0.5051 & 0.5354 & 0.0707 & 0.1515 & 0.7879 & 0.0707 & 0.0606 & 0.8990 \\
\hline 7500 & (f) & 0.5051 & 0.5354 & 0.0707 & 0.1515 & 0.7879 & 0.0707 & 0.0707 & 0.8889 \\
\hline 7500 & (g) & 0.5176 & 0.5333 & 0.0706 & 0.1529 & 0.7922 & 0.0667 & 0.0706 & 0.9098 \\
\hline
\end{tabular}

\subsubsection{First Hopf bifurcation}

A survey of available literature reveals that the precise value of $R e$ at which the first Hopf bifurcation occurs is somewhat contentious, with most current studies (all of which are for incompressible flow) ranging around $R e=7400-8500[9,28,29]$. Here, we do not intend to give a precise value because it is a well observed grid effect that the critical Reynolds number increases (shifts to the right) with refinement (see, e.g., Fig. 3 in Ref. [29]). Rather, we will be content to localise the first bifurcation and, in doing so, demonstrate that limiters are capable of regularising without affecting fundamental flow features.

To localise the first bifurcation we take the following algorithmic approach. Entropic equilibria are in use. The initial uniform fluid density profile is $\rho=1.0$ and the velocity of the lid is $u_{0}=1 / 10$ (in lattice units). We record the unsteady velocity data at a single control point with coordinates $(L / 16,13 L / 16)$ and run the simulation for 5000 non-dimensionless time units $\left(5000 L / u_{0}\right.$ time steps $)$. Let us denote the final $1 \%$ of this signal by $\left(u_{\mathrm{sig}}, v_{\mathrm{sig}}\right)$. We then compute the energy $E_{u}\left(\ell_{2}\right.$-norm normalised by non-dimensional signal duration) of the deviation of $u_{\text {sig }}$ from its mean:

$$
E_{u}:=\left\|\sqrt{\frac{L}{u_{0}\left|u_{\mathrm{sig}}\right|}}\left(u_{\mathrm{sig}}-\overline{u_{\mathrm{sig}}}\right)\right\|_{\ell_{2}},
$$

where $\left|u_{\text {sig }}\right|$ and $\overline{u_{\text {sig }}}$ denote the length and mean of $u_{\text {sig }}$, respectively. We choose this robust statistic instead of attempting to measure signal amplitude because of numerical noise in the LBM simulation. The source of noise in LBM is attributed to the existence of an inherently unavoidable neutral stability direction in the numerical scheme (see, e.g., Ref. [8]).

We opt not to employ the "bounce-back" boundary condition used in the previous steady state study. Instead we will use the diffusive Maxwell boundary condition (see, e.g., Ref. [10]), which was first applied to LBM in Ref. [1]. The essence of the condition is that populations reaching a boundary are reflected, proportional to equilibrium, such that 


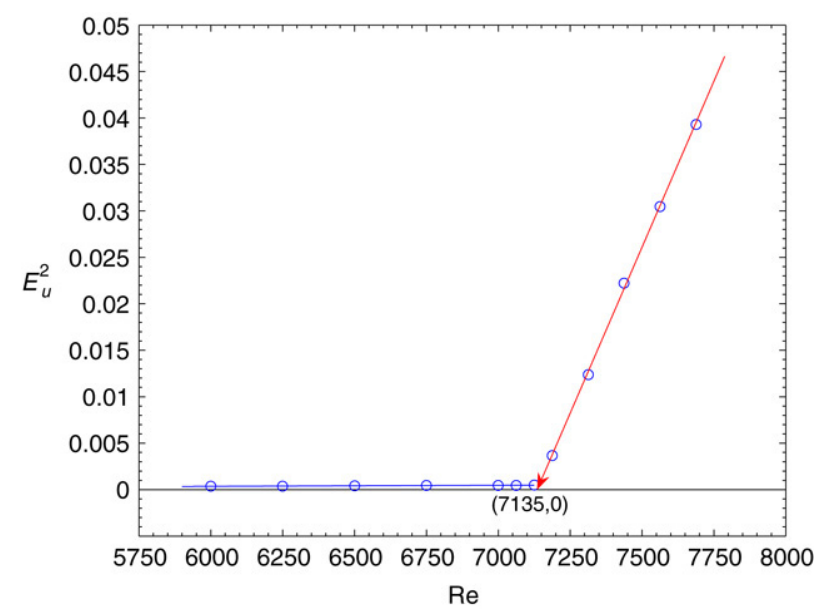

Fig. 8. Plot of energy squared, $E_{u}^{2}(27)$, as a function of Reynolds number, $R e$, using LBGK regularised with the median filter limiter with $\delta=10^{-3}$ on a $100 \times 100$ grid. Straight lines are lines of best fit. The intersection of the sloping line with the $x$-axis occurs close to $R e=7135$.

mass balance (in the bulk) and detailed balance are achieved. The boundary condition coincides with "bounce-back" in each corner of the cavity.

To illustrate, immediately following the advection of populations consider the situation of a wall, aligned with the lattice, moving with velocity $u_{\text {wall }}$ and with outward pointing normal to the wall in the negative $y$-direction (this is the situation on the lid of the cavity with $u_{\text {wall }}=u_{0}$ ). The implementation of the diffusive Maxwell boundary condition at a boundary site $(x, y)$ on this wall consists of the update

$$
f_{i}(x, y, t+1)=\gamma f_{i}^{*}\left(u_{\text {wall }}\right), \quad i=4,7,8,
$$

with

$$
\gamma=\frac{f_{2}(x, y, t)+f_{5}(x, y, t)+f_{6}(x, y, t)}{f_{4}^{*}\left(u_{\text {wall }}\right)+f_{7}^{*}\left(u_{\text {wall }}\right)+f_{8}^{*}\left(u_{\text {wall }}\right)} .
$$

Observe that, because density is a linear factor of the equilibria (21), the density of the wall is inconsequential in the boundary condition and can therefore be taken as unity for convenience. As is usual, only those populations pointing into the fluid at a boundary site are updated. Boundary sites do not undergo the collisional step that the bulk of the sites are subjected to.

We prefer the diffusive boundary condition over the often preferred "bounce-back" boundary condition with constant lid profile. This is because we have experienced difficulty in separating the aforementioned numerical noise from the genuine signal at a single control point using "bounce-back". We remark that the diffusive boundary condition does not prevent unregularised LBGK from failing at some critical Reynolds number $R e>5000$.

Now, we conduct an experiment and record (27) over a range of Reynolds numbers. In each case the median filter limiter is employed with parameter $\delta=10^{-3}$. Since the transition between steady and periodic flow in the lid-driven cavity is known to belong to the class of standard Hopf bifurcations we are assured that $E_{u}^{2} \propto R e$ [15]. Fitting a line of best fit to the resulting data localises the first bifurcation in the lid-driven cavity flow to $R e=7135$ (Fig. 8). This value is within the tolerance of $R e=7402 \pm 4 \%$ given in Ref. [29] for a $100 \times 100$ grid. We also provide a (time averaged) phase space trajectory and Fourier spectrum for $R e=7375$ at the monitoring point (Figs. 9 and 10) which clearly indicate that the first bifurcation has been observed.

\section{Test discussion and a priori estimations of additional dissipation}

The diversity of possible nonequilibrium entropy limiters is huge. The examples provided by our tests present only a tiny fraction of these possibilities. For better orientation in this world one needs some a priori estimates. Of course, the first question should concern additional dissipation. It is easy to find all the dissipation a posteriori (we presented all the necessary techniques in Section 3.4), but for a priori estimates we need some hypotheses about the $\Delta S$ distribution. 


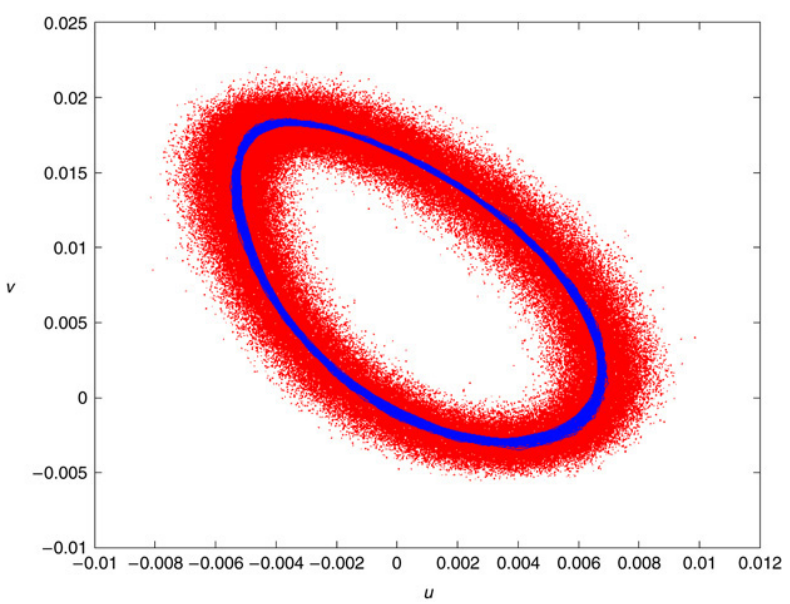

Fig. 9. A phase trajectory for velocity components for the signal $\left(u_{\text {sig }}, v_{\text {sig }}\right)$ at the monitoring point $(L / 16,13 L / 16)$ using LBGK regularised with the median filter limiter with $\delta=10^{-3}$ on a $100 \times 100$ grid $(R e=7375)$. Dots represent simulation results at various time moments and the solid line is a 100 step time average of the signal.

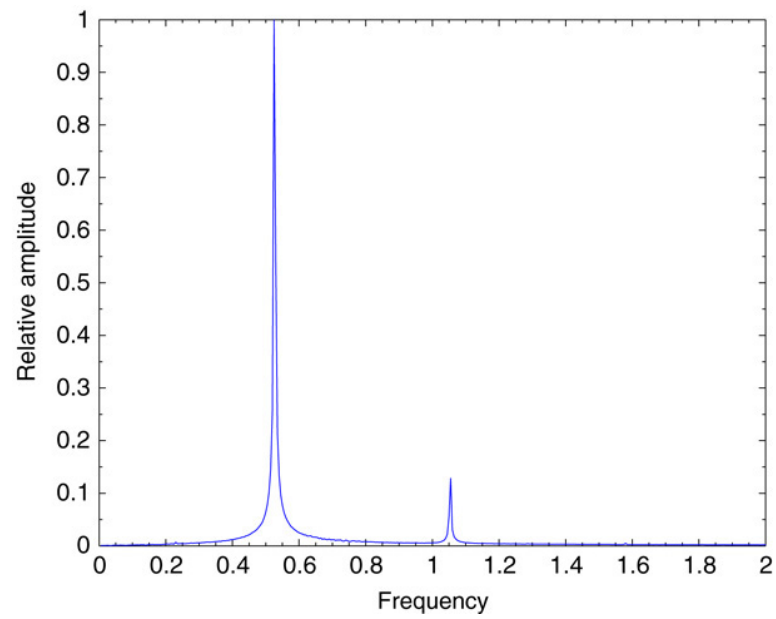

Fig. 10. Relative amplitude spectrum for the signal $u_{\text {sig }}$ at the monitoring point $(L / 16,13 L / 16)$ using LBGK regularised with the median filter limiter with $\delta=10^{-3}$ on a $100 \times 100$ grid $(R e=7375)$. We measure a dominant frequency of $\omega=0.525$.

Let us start from the most straightforward idea: estimation at the average. Let us take $\Delta S=\mathrm{E}(\Delta S)$ and estimate the additional dissipation. This corresponds to the hypothesis that $\Delta S$ is distributed near its mean value with relatively small deviation. Immediately, we find that under this assumption any local limiter of the form (10) is simply equivalent to multiplying $f-f^{*}$ by $\phi(\mathrm{E}(\Delta S))$ and it leads to additional viscosity $\Delta v \sim(1-\phi(\mathrm{E}(\Delta S)))$ in dimensionless form. However, as we can see from Figs. 4 and 5, for example, performance can be significantly different for limiters that have the same $\phi(\mathrm{E}(\Delta S))$. This implies that the hypothesis about a narrow distribution of $\Delta S$ near its mean value does not work, and performance of a limiter depends on the value of $\phi(\Delta S)$ for larger $\Delta S$.

There is a practical consequence of this observation too. A limiter's work is important for $\Delta S>\mathrm{E}(\Delta S)$ (or even $\Delta S \gg \mathrm{E}(\Delta S))$. Hence, all the influence of a limiter on the dynamics at $\Delta S \sim \mathrm{E}(\Delta S)$ is a parasitic effect, and this should be excluded. Therefore, for smooth limiters, $\phi(\Delta S)$, it is natural to assume that $\phi(0)=1$ and $\phi^{\prime}(0)=0$. This means that, for small $\Delta S$, collisions with a limiter coincide with LBM collisions in the first two orders.

The first attempt to improve the hypothesis about the distribution of $\Delta S$ is an assumption that $\Delta S$ has exponential distribution with probability density

$$
p(\Delta S)=\frac{1}{\mathrm{E}(\Delta S)} \exp (-\Delta S / \mathrm{E}(\Delta S)) .
$$


This distribution for entropy seems very familiar from equilibrium statistical physics, but the tests of the Ehrenfests' limiters [6,7] and of threshold limiters (Fig. 6) show that even a limiter with a threshold $\sim 20 \mathrm{E}(\Delta S)$ significantly improves the picture. For the exponential distribution, $\mathrm{P}(E>20 \mathrm{E}(\Delta S))=\exp (-20)$ : on our grid, the appearance of such points is improbable.

A dynamical reason for fat tails can be a so-called superstatistics [3], where one has a superposition of local simple distributions whose parameters fluctuate on a rather large spatio-temporal scale. The idea of superstatistics can help us: the domain of flow can be split into several subdomains, in any of these subdomains there is an exponential distribution of $\Delta S$ and as a result there is a mixed "superdistribution" in the whole domain.

Already two exponential distributions give a much more realistic picture:

$$
p(\Delta S)=\frac{1-q}{\overline{\Delta S}_{1}} \exp \left(-\Delta S / \overline{\Delta S}_{1}\right)+\frac{q}{\overline{\Delta S}_{2}} \exp \left(-\Delta S / \overline{\Delta S}_{2}\right)
$$

with $q \ll 1$ and $\overline{\Delta S}_{1} \ll \overline{\Delta S}_{2}$. For this distribution, the threshold limiter (and other limiters that do not affect the average points with $\Delta S \sim \mathrm{E}(\Delta S))$ acts on the points that belong to the second distribution, far from the $\Delta S$ mean value. This point of view is supported by the following observation: the entropy productions for all successfully working limiters in the shock tube test (Section 4.3) are almost the same, even if the control parameter $\delta$ changes by several orders of magnitude. Of course, this observation also supports other hypotheses with a representation of $p(\Delta S)$ as a mixture of two simple distributions with significantly different $\mathrm{E}(\Delta S)$. For example, if $p(\Delta S)=$ $(1-q) \delta\left(\Delta S-\overline{\Delta S}_{1}\right)+q \delta\left(\Delta S-\overline{\Delta S}_{2}\right)$ with $q \ll 1$ and $\overline{\Delta S}_{1} \ll \overline{\Delta S}_{2}$, then the behaviour of the dissipation will also be similar.

Again, we can extract a practical consequence. It is not necessary to apply limiters at all points. In addition to a threshold in $\Delta S$ we can also use a threshold number of points $k$ and apply limiters at not more than $k$ points with maximal $\Delta S$. For example, if we apply the Ehrenfests' or the threshold limiter only at one point of the shock profile, then it changes the picture drastically: there remain some fluctuations, but their amplitude decreases by orders [6,7].

Let us estimate the additional dissipation produced by the threshold limiter for the nonequilibrium entropy distribution (28). If the threshold value $\Delta S_{\mathrm{t}} \gg \overline{\Delta S_{1}}$ and the allowed number of points for the limiter action $k<q N$, where $N$ is the total number of points, then only the second exponential distribution affects the limiter work. A simple explicit estimation gives us the following:

If $q N \exp \left(-\Delta S_{\mathrm{t}} / \overline{\Delta S_{2}}\right)>k$ then we expect the number of points with application of the limiter to be $\sim k$ and the average dissipation per such point to be

$$
S_{\mathrm{pp}} \approx \int_{\overline{\Delta S_{2}} \ln (q N / k)}^{\infty} \frac{\Delta S-\Delta S_{\mathrm{t}}}{\overline{\Delta S_{2}}} \exp \left(\frac{-\Delta S}{\overline{\Delta S_{2}}}\right) \mathrm{d} \Delta S=\overline{\Delta S_{2}} \frac{k}{q N}\left[\ln \frac{q N}{k}-\frac{\Delta S_{\mathrm{t}}}{\overline{\Delta S_{2}}}+1\right],
$$

or $k S_{\mathrm{pp}}$ totally.

If $q N \exp \left(-\Delta S_{\mathrm{t}} / \overline{\Delta S_{2}}\right)<k$ then we expect the number of points with application of the limiter to be $\sim q N \exp \left(-\Delta S_{\mathrm{t}} / \overline{\Delta S_{2}}\right)<k$ and the average dissipation per such point to be

$$
S_{\mathrm{pp}} \approx \int_{\Delta S_{\mathrm{t}}}^{\infty} \frac{\Delta S-\Delta S_{\mathrm{t}}}{\overline{\Delta S_{2}}} \exp \left(\frac{-\Delta S}{\overline{\Delta S_{2}}}\right) \mathrm{d} \Delta S=\overline{\Delta S_{2}} \exp \left(\frac{-\Delta S_{\mathrm{t}}}{\overline{\Delta S_{2}}}\right),
$$

or $q N \overline{\Delta S_{2}} \exp \left(-2 \Delta S_{\mathrm{t}} / \overline{\Delta S_{2}}\right)$ in total.

These estimates use some features of the $\Delta S$ distribution. Any theoretical or computational study of this distribution immediately gives us a clue for estimates of a limiter's work.

In the last case, we can estimate additional viscosity in the highly nonequilibrium region that corresponds to the second exponent in the distribution (28). Indeed, the number of points in this region is $q N$, the average $\Delta S$ is $\overline{\Delta S_{2}}$, additional dissipation per point in this region is $\overline{\Delta S_{2}} \exp \left(-2 \Delta S_{\mathrm{t}} / \overline{\Delta S_{2}}\right)$. This corresponds to additional contraction to equilibrium with coefficient $\sim 1-\frac{1}{2} \exp \left(-2 \Delta S_{\mathrm{t}} / \overline{\Delta S_{2}}\right)$ per collision step. Due to standard LBGK estimates, the correspondent additional viscosity is $\Delta v \approx \frac{1}{4} \exp \left(-2 \Delta S_{\mathrm{t}} / \overline{\Delta S_{2}}\right)<\frac{k^{2}}{4(q N)^{2}}$ (in dimensionless units). For example, for $\Delta v \leq 0.01$ this gives $\Delta S_{\mathrm{t}} \gtrsim 1.6 \overline{\Delta S_{2}}$, for $\Delta v \leq 0.001$ we have $\Delta S_{\mathrm{t}} \gtrsim 2.8 \overline{\Delta S_{2}}$, and for $\Delta v \leq 0.0001$ we have $\Delta S_{\mathrm{t}} \gtrsim 3.9 \overline{\Delta S_{2}}$.

Does the distribution of $\Delta S$ have a long tail? To support this hypothesis we computed the histograms of $\Delta S$ (Fig. 11) for the experiment presented in Fig. 3. It is straightforward to estimate that such a long tail is practically 

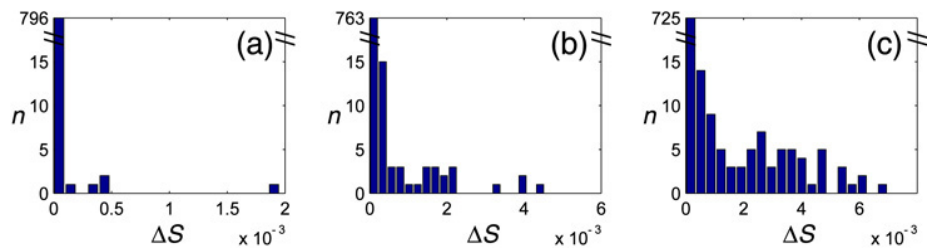

Fig. 11. Histograms of nonequilibrium entropy $\Delta S$ for the $1: 2$ athermal shock tube simulation with (a) $v=0.066$, (b) $v=0.0066$ and (c) $v=0.00066$ after 400 time steps using LBGK (23) without any limiter. Entropic equilibria (18) with perfect entropy (17) are used. We use a broken $y$-axis. These histograms correspond to computations represented on Fig. 3(a),(b),(c). The $x$-axis interval is from zero to (a) $450 \mathrm{E}(\Delta S)$, (b) $97 \mathrm{E}(\Delta S)$ and (c) $32 \mathrm{E}(\Delta S)$, respectively. It is divided into 20 bins.

impossible for an exponential distribution. For example, the probability of having by chance the maximal $\Delta S$ so far from $\mathrm{E}(\Delta S)$ (for 801 points) is $p<801 \exp \left(-\Delta S_{\max } / \mathrm{E}(\Delta S)\right.$ ). For the histograms presented in Fig. 11, we get: (a) $p<10^{-170}$, (b) $p<10^{-21}$ and (c) $p<10^{-8}$, respectively. Of course, the probability of appearance of the whole tails by chance in cases (b) and (c) is much smaller.

Direct estimation of additional dissipation for the median filter limiter is impossible without hypotheses about pair correlations in the $\Delta S$ field. Computational tests for shock tubes and for lid-driven cavity flow show that the median filter limiter (applied to the "salt" noise only when $\Delta S$ exceeds the median value, and, moreover when the value of the difference between the current value and the median value is higher than a given threshold) works properly both for shocks and for $2 \mathrm{D}$ flow with $R e \lesssim 8000$ on a $100 \times 100$ grid without any sign of parasitic viscosity. Additional entropy production for the median filter limiters in shock tube tests is essentially the same as for other successful limiters (it is 10\%-20\% less; see Fig. 7), but the value of nonequilibrium entropy $\Delta S$ for the median limiter is much higher than for local value limiters, and small (macroscopically unobservable) oscillations of $\Delta S$ appear. The distribution of $\Delta S$ is much more sensitive to the choice of limiter than the additional dissipation. This is one more practical hint from the tests performed. This indicator shows that the median filter disturbs the original LBGK kinetics less than other tested limiters with the same or sometimes better stabilisation results.

All these observations and successful tests allow us to suggest the minimal median filter limiter as a "limiter of choice". There is one important addition that we did not test in this work, which improves performance of the median limiter: one should not apply the limiter at all points where the threshold is exceeded, but at a fixed number of such points (not more than a given $k$, exactly as we did for the Ehrenfests' limiters [7]).

\section{Conclusions}

We have constructed a system of nonequilibrium entropy limiters for the LBM:

- the positivity rule that provides positivity of distribution;

- the pointwise entropy limiters based on selection and correction of most nonequilibrium values;

- filters of nonequilibrium entropy, and the median filter as a filter of choice.

All these limiters exploit physical properties of LBM and allow control of total additional entropy production. In general, they do the same work for LBM as flux limiters do for finite differences, finite volumes and finite elements methods, and come into operation when sharp gradients are present. For smoothly changing waves, the limiters do not operate and the spatial derivatives can be represented by higher order approximations without introducing non-physical oscillations. But there are some differences too: for LBM the main idea behind the construction of nonequilibrium entropy limiter schemes is to limit a scalar quantity - the nonequilibrium entropy - or to delete the "salt and pepper" noise from the field of this quantity. We do not touch the vectors or tensors of spatial derivatives, as one does for flux limiters.

Standard test examples demonstrate that the limiters developed erase spurious oscillations without blurring of shocks, and do not affect smooth solutions. The limiters that we have tested do not produce a noticeable additional dissipation and allow us to reproduce the first Hopf bifurcation for a 2D lid-driven cavity on a coarse $100 \times 100$ grid. At the same time the simplest median filter deletes the spurious post-shock oscillations for low viscosity.

Perhaps it is impossible to find one best nonequilibrium entropy limiter for all problems. It is a special task to construct the optimal limiters for a specific class of problems. 


\section{Acknowledgments}

Discussion of the preliminary version of this work with S. Succi and participants of the lattice Boltzmann workshop held on 15th November 2006 in Leicester (UK) was very important. Author A.N. Gorban is grateful to S.K. Godunov for the course of numerical methods given many years ago at Novosibirsk University. This work was supported by Engineering and Physical Sciences Research Council (EPSRC) grant number GR/S95572/01.

\section{References}

[1] S. Ansumali, I.V. Karlin, Kinetic boundary conditions in the lattice Boltzmann method, Phys. Rev. E 66 (2002) 026311.

[2] S. Ansumali S, I.V. Karlin, H.C. Ottinger, Minimal entropic kinetic models for hydrodynamics, Europhys. Lett. 63 (2003) $798-804$.

[3] C. Beck, Stretched exponentials from superstatistics, Physica A 365 (2006) 96-101.

[4] R. Benzi, S. Succi, M. Vergassola, The lattice Boltzmann-equation — theory and applications, Phys. Rep. 222 (1992) $145-197$.

[5] B.M. Boghosian, J. Yepez, P.V. Coveney, A.J. Wager, Entropic lattice Boltzmann methods, R. Soc. Lond. Proc. Ser. A Math. Phys. Eng. Sci. 457 (2001) 717-766.

[6] R.A. Brownlee, A.N. Gorban, J. Levesley, Stabilisation of the lattice-Boltzmann method using the Ehrenfests' coarse-graining, condmat/0605359, 2006.

[7] R.A. Brownlee, A.N. Gorban, J. Levesley, Stabilisation of the lattice-Boltzmann method using the Ehrenfests' coarse-graining, Phys. Rev. E 74 (2006) 037703.

[8] R.A. Brownlee, A.N. Gorban, J. Levesley, Stability and stabilization of the lattice Boltzmann method, Phys. Rev. E 75 (2007) 036711.

[9] C.-H. Bruneau, M. Saad, The 2D lid-driven cavity problem revisited, Comput. Fluids 35 (2006) 326-348.

[10] C. Cercignani, Theory and Application of the Boltzmann Equation, Scottish Academic Press, Edinburgh, 1975.

[11] S.R. Chatkravathy, S. Osher, High resolution applications of the Osher upwind scheme for the Euler equations, AIAA Paper 83-1943, In: Proc. AIAA 6th Computational Fluid Dynamics Conference, 1983, p. 363-373.

[12] S. Chen, G.D. Doolen, Lattice Boltzmann method for fluid flows, Annu. Rev. Fluid. Mech. 30 (1998) 329-364.

[13] A.J. Chorin, O.H. Hald, R. Kupferman, Optimal prediction with memory, Physica D 166 (2002) 239-257.

[14] P. Ehrenfest, T. Ehrenfest, The Conceptual Foundations of the Statistical Approach in Mechanics, Dover Publications Inc., New York, 1990.

[15] N.K. Ghaddar, K.Z. Korczak, B.B. Mikic, A.T. Patera, Numerical investigation of incompressible flow in grooved channels. Part 1. Stability and self-sustained oscillations, J. Fluid Mech. 163 (1986) 99-127.

[16] S.K. Godunov, A difference scheme for numerical solution of discontinuous solution of hydrodynamic equations, Math. Sbornik 47 (1959) 271-306.

[17] A.N. Gorban, Equilibrium encircling, in: Equations of Chemical Kinetics and their Thermodynamic Analysis, Nauka, Novosibirsk, 1984.

[18] A.N. Gorban, I.V. Karlin, H.C. Öttinger, L.L. Tatarinova, Ehrenfest's argument extended to a formalism of nonequilibrium thermodynamics, Phys. Rev. E 62 (2001) 066124.

[19] A.N. Gorban, Basic types of coarse-graining, in: A.N. Gorban, N. Kazantzis, I.G. Kevrekidis, H.-C. Öttinger, C. Theodoropoulos (Eds.), Model Reduction and Coarse-Graining Approaches for Multiscale Phenomena, Springer, Berlin, Heidelberg, New York, 2006, pp. 117-176. cond-mat/0602024.

[20] F. Higuera, S. Succi, R. Benzi, Lattice gas - dynamics with enhanced collisions, Europhys. Lett. 9 (1989) 345-349.

[21] S. Hou, Q. Zou, S. Chen, G. Doolen, A.C. Cogley, Simulation of cavity flow by the lattice Boltzmann method, J. Comput. Phys. 118 (1995) 329-347.

[22] I.V. Karlin, A.N. Gorban, S. Succi, V. Boffi, Phys. Rev. Lett. 81 (1998) 6-9.

[23] I.V. Karlin, A. Ferrante, H.C. Öttinger, Perfect entropy functions of the lattice Boltzmann method, Europhys. Lett. 47 (1999) $182-188$.

[24] I.V. Karlin, S. Ansumali, C.E. Frouzakis, S.S. Chikatamarla, Elements of the lattice Boltzmann method I: Linear advection equation, Commun. Comput. Phys. 1 (2006) 616-655.

[25] S. Kullback, Information Theory and Statistics, Wiley, New York, 1959.

[26] C.D. Levermore, J.-G. Liu, Oscillations arising in numerical experiments, Physica D 99 (1996) 191-216.

[27] Y. Li, R. Shock, R. Zhang, H. Chen, Numerical study of flow past an impulsively started cylinder by the lattice-Boltzmann method, J. Fluid Mech. 519 (2004) 273-300.

[28] T.W. Pan, R. Glowinksi, A projection/wave-like equation method for the numerical simulation of incompressible viscous fluid flow modeled by the Navier-Stokes equations, Comput. Fluid Dyn. J. 9 (2000) 28-42.

[29] Y.-F. Peng, Y.-H. Shiau, R.R. Hwang, Transition in a 2-D lid-driven cavity flow, Comput. Fluids 32 (2003) 337-352.

[30] W.K. Pratt, Digital Image Processing, Wiley, New York, 1978.

[31] H. Qian, Relative entropy: free energy associated with equilibrium fluctuations and nonequilibrium deviations, Phys. Rev. E. 63 (2001) 042103.

[32] P.L. Roe, Characteristic-based schemes for the Euler equations, Ann. Rev. Fluid Mech. 18 (1986) 337-365.

[33] S. Succi, The Lattice Boltzmann Equation for Fluid Dynamics and Beyond, Oxford University Press, New York, 2001.

[34] S. Succi, I.V. Karlin, H. Chen, Role of the H theorem in lattice Boltzmann hydrodynamic simulations, Rev. Mod. Phys. 74 (2002) 1203-1220.

[35] P.K. Sweby, High resolution schemes using flux-limiters for hyperbolic conservation laws, SIAM J. Num. Anal. 21 (1984) $995-1011$.

[36] E. Tadmor, W. Zhong, Entropy stable approximations of Navier-Stokes equations with no artificial numerical viscosity, J. Hyperbolic Differ. Equ. 3 (2006) 529-559. 
[37] F. Tosi, S. Ubertini, S. Succi, H. Chen, I.V. Karlin, Numerical stability of entropic versus positivity-enforcing lattice Boltzmann schemes, Math. Comput. Simulation 72 (2006) 227-231.

[38] B. Van Leer, Towards the ultimate conservative difference scheme III. Upstream-centered finite-difference schemes for ideal compressible flow, J. Comput. Phys. 23 (1977) 263-275.

[39] P. Wesseling, Principles of Computational Fluid Dynamics, in: Springer Series in Computational Mathematics, vol. 29, Springer, Berlin, 2001. 